\title{
Effective Capture of Non-Graspable Objects for Space Robots Using Geometric Cage Pairs
}

\author{
Xin Zhang, Student Member, IEEE, Jinguo Liu, Senior Member, IEEE, Jingkai Feng, \\ Yuwang Liu, Member, IEEE, and Zhaojie Ju, Senior Member, IEEE
}

\begin{abstract}
Capture and removal of space debris is challenging in robotic on-orbit servicing (OOS) activities. A large portion of space debris does not possess any graspable features, which makes conventional grippers inapplicable. To handle such non-graspable objects, a space robotic capture system is presented. A dual-arm space robot simulator that has the advantages of miniaturization and scalability is designed for ground tests. Inspired by robotic caging, we propose a novel capture method that uses a series of hollow-shaped end-effector pairs to cage the antipodal pairs of non-graspable objects. To apply the caging-pair method steadily, space robots need exerting a squeezing action on objects, which can be characterized by the motion and force manipulation of two robotic arms in the assigned directions. Based on the velocity and force manipulability transmission ratios, a caging compatibility index is proposed to describe the capturing ability in this manner. Via the optimization of the desired caging compatibility index, an effective algorithm is proposed to plan near-optimal joint configurations for pre-grasping cages. Finally, both simulation studies and experimental tests are conducted to evaluate the performance of the proposed capture method.
\end{abstract}

Index Terms -Non-graspable objects, space robot simulator, caging-pair method, caging compatibility index.

\section{INTRODUCTION}

$\mathrm{R}$ ECENTLY, the gradual accumulation of space debris has increased the probability of collisions between debris and on-orbit satellites. This threatens the long-term security and stability of the space environment. Active debris removal (ADR) has become an essential task for the entire spaceflight community [1]. Most space debris comes from abandoned rocket bodies, defunct satellites, their exploded fragments, space rocks, and out-of-control spacecraft, which are typically called non-cooperative objects [2]. The development of space robotic technologies for dealing with non-cooperative objects has attracted substantial interest [3].

Several OOS projects, for example, the Phoenix program and Robotic Servicing of Geosynchronous Satellites (RSGS), have emphasized the consideration of non-cooperative objects [4] as they exhibit numerous uncertainties in shape and size. In this study, from the point of implementing grasping using conventional grippers, we classify space objects into graspable and non-graspable objects (typical examples are presented in Figs. 1(a) and 1(b), respectively). Graspable objects have graspable fixtures, whereas non-graspable objects do not possess such graspable features and cannot be grasped by conventional grippers. To avoid ambiguity, grasping is distinguished from capturing. Capturing includes a wide range of strategies for controlling the mobility of an object [2], whereas grasping is to hold an object firmly, which is one of many capturing methods. In current, most studies focus on capturing and stabilizing graspable objects, and their graspable features include payload adapter rings [5], engine nozzle cones [6], solar panel brackets [7], and other customized grapple fixtures [8], [9]. Yoshida et al. [6] proposed an impedance matching method for capturing a simulated satellite. Moosavian et al. [10] proposed a multiple impedance control for a dual-arm system to manipulate a cooperative object. Aghihi [11] proposed an optimal estimation and planning method for capturing tumbling objects. Mccourt and Silva [12] proposed a constrained predictive control strategy for capturing a spinning simulated satellite. Huang et al. [13] proposed a spacecraft attitude takeover control scheme for stabilizing graspable objects in the post-grasping phase. For these capture tasks [5]-[13], the research objects are graspable objects and robotic arms and grippers are common tools. In addition, universal grippers based on the jamming of granular material [14] or underactuated fingers [15], [16] have been developed for realizing the adaptive capture of irregular objects. Due to the vacuum and high-temperature-difference environment and practical factors such as weight and volume restriction requirements, these methods [14]-[16] are impracticable for grasping non-graspable objects in space [17]. For dealing with this class of objects, two main strategies are employed: One is to use flexible capturing theories and methods, including tether nets [18], [19], tether grippers [20], [21], robotic capsules [22] and robotic tentacles [23]; the other is to capture objects using robotic arms with customized capture effectors, of which end-effectors are the most important tools as they can directly determine the success of tasks [24]. An innovative strategy is proposed for adhering directly to the surfaces of objects and an electro-adhesive gripper [17] and a gecko-inspired adhesive gripper [3] are developed for capturing non-graspable objects. 


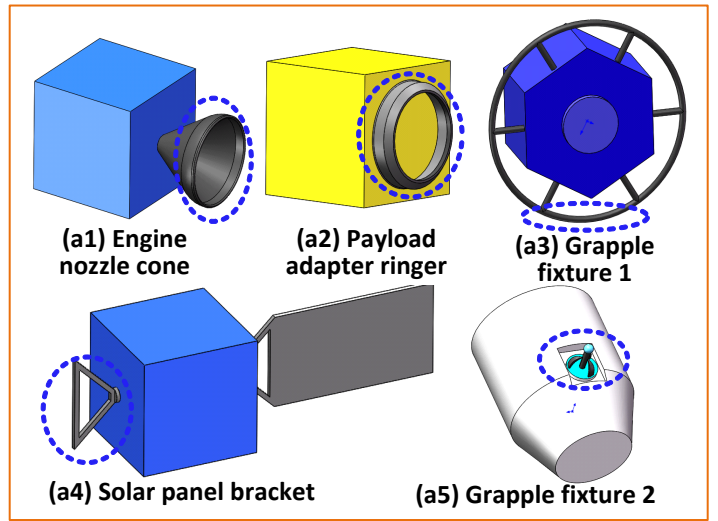

(a) Graspable objects

Fig. 1. Classification of space objects

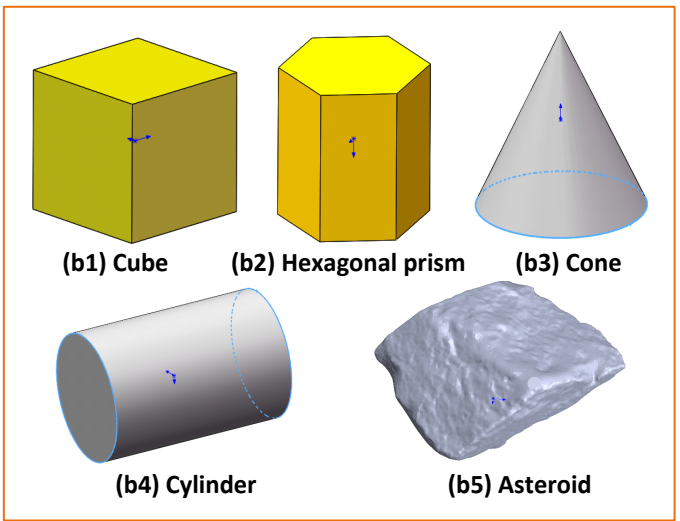

(b) Non-graspable objects
A microspine effector [22] is proposed for capturing asteroids.

The flexible methods are considered attractive solutions because they are adaptive for graspable and non-graspable objects, regardless of shape features. However, in practice, flexible capturing methods [18]-[21] are not easy to implement. The main challenge in using tether nets and grippers is to properly fold, release, and control them because their flexible materials easily become entangled, which may result in the failure of the entire mission. Their low reusability is another intrinsic weakness and cannot be ignored. The main drawbacks of the robotic tentacle [23] are its limited manipulability and low reliability. The main preconditions for using the adhesive grippers [3], [17] are that objects must have smooth and flat surfaces to ensure a large contact area and adhesive force, and they are susceptible to the space environment. For the two methods in [22], the underlying strategy of the capture effector is to form robotic cages for capturing various objects; however, the unit-type cage is accompanied by limited caging volume and manipulability. The main objective of the caging process is to confine an object within a region, similar to a bird cage; it is a purely geometric method without the force analysis [25].

In summary, for practical applications, capturing methods are desired to satisfy the following requirements: 1) reliability, to guarantee the performance of the capturing tools in implementing and executing the capture tasks; 2) robustness, to overcome the uncertainties from objects and the space environment; and 3) reusability, to ensure that the capturing devices can be reused many times.

As we stated earlier, non-graspable objects are of various shapes and sizes and there is no universal method for dealing with all types of non-graspable objects. In Fig. 1(b), some typical objects with convex hulls are selected as our simulated objects like satellite structures, space rocks, and et al. Inspired by robotic caging capture, we propose a novel caging-pair method that adopts a series of geometric effector pairs to form combined robotic cages for capturing non-graspable objects. Compared with the unit-type caging method [22], the caging-pair method has more dexterous manipulation ability. In addition, based on the principle of geometric constraints, the proposed method is intrinsically robust and reliable in dealing with shape and environment uncertainties. The main contributions of this study are summarized as follows:
1) A caging-pair method that uses a series of hollow-shaped end-effector pairs is proposed to form the combined type of robotic cages for capturing non-graspable objects.

2) A free-flying space robot simulator is developed to evaluate the proposed method, which has the advantage of scalability for changing joint modules and end-effectors.

3) Based on the concept of the task compatibility, we propose a caging compatibility index to describe the capturing capability of the caging-pair method.

4) Based on the caging compatibility, an effective algorithm is proposed for planning the joint configuration of pre-grasping cages to realize a desired robotic cage.

The remainder of this paper is organized as follows: Section II describes the system design of the simulator. Section III analyzes the kinematics and statics of the dual-arm space robot. Then, the caging-pair method, the end-effector design, and the caging compatibility index are introduced in Section IV. In Section V, the planning algorithm, simulation studies, and experiments are conducted to evaluate the proposed method. Finally, the conclusions are presented in Section VI.

\section{Space Robot Simulator Design}

With the development of space robotics, researchers have focused on dual- and multi-arm space robots because of their dexterous manipulation capabilities [4]. In general, for space robots, there are two working modes: free-flying mode (both the position and attitude of the base are actively controlled) and free-floating mode (neither of them is controlled) [26]. Although the free-floating mode has the fuel-saving advantage, the dynamic coupling and singularity complicate the trajectory planning and control of free-floating space robots [27]. In consideration of the operational accuracy and safety for space tasks, the bases of free-flying space robots can be controlled to stay still. Thus, we aim at developing a dual-arm free-flying simulator for ground tests.

\section{A. Related Work}

Space robots operate in the microgravity atmosphere and some research institutes have developed various simulators in the past decades. For example, a neutral-underwater-buoyancy simulator was developed in [28], active and passive suspension systems were designed in [29], [30], hardware-in-the-loop systems were proposed in [9] and [31], 


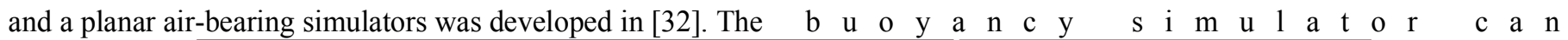

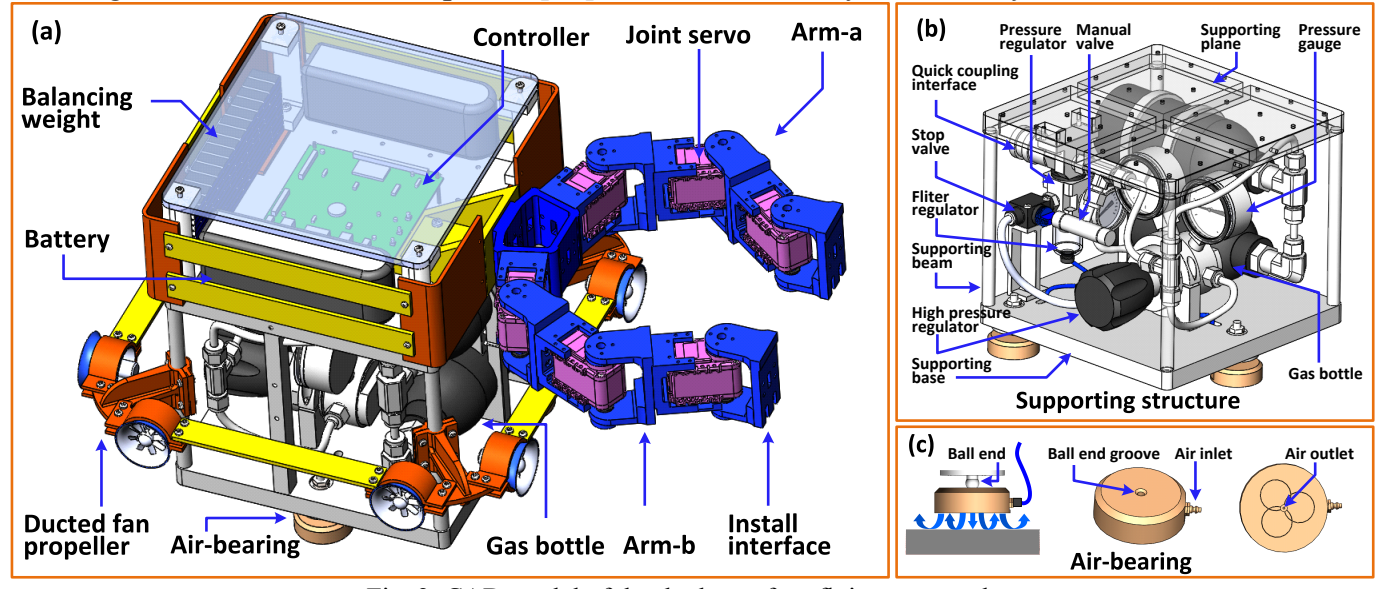

Fig. 2. CAD model of the dual-arm free-flying space robot

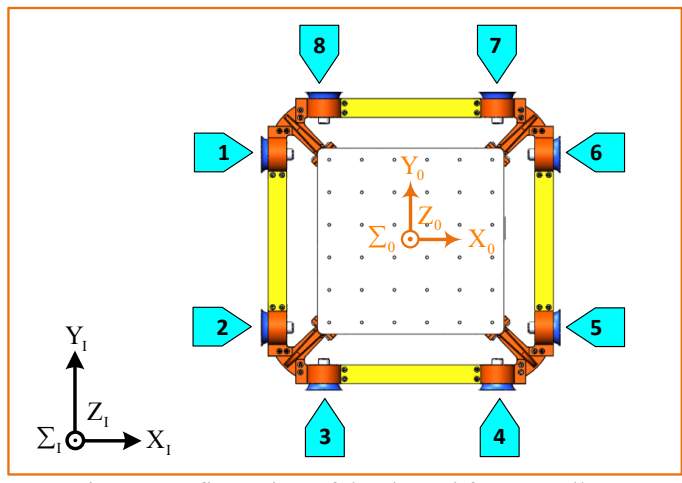

Fig. 3. Configuration of the ducted fan propellers

simulate 3D motion; however, it causes viscous resistance of water and incurs high maintenance costs. Similarly, suspension simulators cause frictional resistance and lead to low simulation accuracy. The hardware-in-the-loop method can realize high accuracy; however, it cannot authentically simulate the dynamic properties of space robots. Although the planar air-bearing simulators can only simulate 2D motion, they generate low frictional resistance and can realize high accuracy. Considering various factors (including accuracy, maintenance costs, and occupied area), we develop an air-bearing simulator [33] with scalability under laboratory conditions. The platform includes three sub-systems: the mechanical structure design (Figs. 2 and 3), the pneumatic system (Fig. 4), and the electronic system (Fig. 5). The corresponding details are introduced as follows.

\section{B. Structural Design of the Air-Bearing Simulator}

As illustrated in Fig. 2(a), the dual-arm free-flying space robot simulator includes a main supporting structure, eight ducted fan propellers, and two 3-degree-of-freedom (3-DOF) modular robotic arms (arm-a and arm-b). The supporting structure includes a supporting base, three air bearings, four supporting beams, and a supporting plane. It has the shape of a cubic frame $\left(200 \times 200 \times 200 \mathrm{~mm}^{3}\right)$. The control components and two arms are fixed on the supporting plane and pneumatic components (in Fig. 2(b)) are fixed on the supporting base. The supporting base is fixed on three air bearings, in which the ball end and ball end groove will achieve an automatic centering effect between the supporting base and air bearings.
As illustrated in Fig. 2(c), three circular grooves are set on the

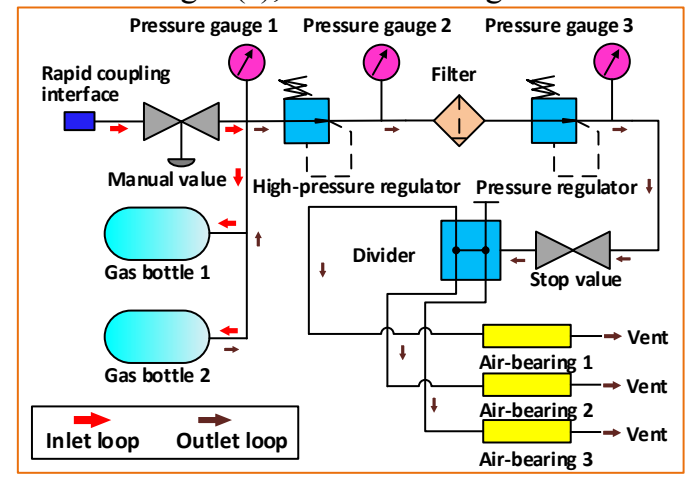

Fig. 4. Pneumatic system design

bottom of each air bearing. When the outlet pressure exceeds $0.3 \mathrm{MPa}$, an air film $(5 \sim 10 \mu \mathrm{m})$ is generated between the air bearings and the granite table to keep the entire system floating freely. Furthermore, as illustrated in Fig. 3, eight ducted fan propellers are orthogonally installed on the supporting beams to realize the decoupling free-flying motion. Here, the propellers on the same side can produce the translational motion (e.g., Propellers 1 and 2 can generate translational thrust in the direction of $\mathrm{X}_{0}$ ) and the propellers on the diagonal can produce the rotating motion (e.g., Propellers 3 and 7 can generate rotating torque around $Z_{0}$ ).

\section{Pneumatic System}

As illustrated in Fig. 4, the pneumatic system contains two loops: an inlet loop and an outlet loop. For the inlet loop, the manual valve should be switched on, and the high-pressure regulator, the pressure regulator, and the stop valve should be turned off. When the rapid coupling interface is connected with a high-pressure nitrogen $\left(\mathrm{N}_{2}\right)$ source, the high-pressure $\mathrm{N}_{2}$ will be transferred to gas bottles via the manual valve. The volume and nominal working pressure of each gas bottle are $0.22 \mathrm{~L}$ and $10 \mathrm{MPa}$, respectively. When gas bottles are filled with $\mathrm{N}_{2}$, the manual valve should be turned off. Pressure gauge 1 is used to indicate the pressure of gas bottles. For the outlet loop, the manual valve and two pressure regulators should be turned off and the stop valve should be switched on. Then, the high-pressure $\mathrm{N}_{2}$ in gas bottles will pass through the high-pressure regulator, the filter, the pressure regulator, and 
the stop valve and, finally, reach the three-branch divider. The pressure of the high-pressure $\mathrm{N}_{2}$ will be reduced to $0.6 \mathrm{MPa}$

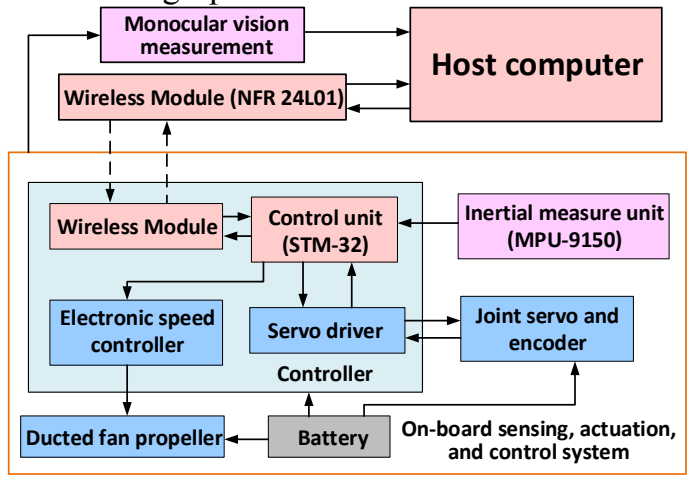

Fig. 5. Electronic system design

the high-pressure regulator and, subsequently, to $0.3 \mathrm{MPa}$ via the second pressure regulator. At last, the low-pressure $\mathrm{N}_{2}$ will be sent to air-bearings, thereby generating the air film between air-bearings and the granite table to support the simulator.

\section{Electronic System}

As illustrated in Fig. 5, the electronic system includes sensing, actuation, and control units. For the control unit, a host computer performs the task planning and an ARM microcontroller (STM-32) is used for the onboard closed-loop feedback control. For the sensor feedback, a monocular camera is used to measure the position information of the simulator and an inertial measurement unit (IMU, MPU-9150) is used to measure the attitude information. Digital servos can be programmed to control robotic arms and ducted fan propellers can be controlled via the pulse-width modulation technique. Wireless modules (NFR 24L01) are used for the signal transmission between the host computer and the onboard controller, and lithium batteries power the simulator.

\section{FREE-FLYING SPACE ROBOT MODELING}

In this section, we will establish and analyze the kinematic and static force models of the proposed dual-arm simulator in free-flying mode. Moreover, considering the dynamic coupling effect, the base of the simulator is controlled to stay still when robotic arms are executing the capture task.

As illustrated in Fig. 6, the proposed simulator is composed of a base and two arms (arm-a and arm-b). Symbols and variables are defined in Table I. Superscript $(\cdot)^{k}$ indicates that symbols and variables are defined for arm- $k$ (in Fig. 6, $k=\{\mathrm{a}, \mathrm{b}\})$; unless otherwise specified, all the symbols and variables are defined in inertial frame $\Sigma_{1}$.

\section{A. Velocity Kinematics Analysis}

For any two vectors, there exists a cross-product equation: $\boldsymbol{k} \times \boldsymbol{a}=\tilde{\boldsymbol{k}} \boldsymbol{a}$. For $\boldsymbol{k}=\left[\begin{array}{ll}k_{\mathrm{x}} & k_{\mathrm{y}} \\ k_{\mathrm{z}}\end{array}\right]^{\mathrm{T}}, \tilde{\boldsymbol{k}}$ is defined as

$$
\tilde{\boldsymbol{k}}=\left[\begin{array}{ccc}
0 & -k_{\mathrm{z}} & k_{\mathrm{y}} \\
k_{\mathrm{z}} & 0 & -k_{\mathrm{x}} \\
-k_{\mathrm{y}} & k_{\mathrm{x}} & 0
\end{array}\right]
$$

In Fig. 6, two position relationships can be obtained as

$$
\boldsymbol{r}_{i}^{k}=\boldsymbol{r}_{0}+\boldsymbol{b}_{0}^{k}+\sum_{j=1}^{i-1}\left(\boldsymbol{a}_{j}^{k}+\boldsymbol{b}_{j}^{k}\right)+\boldsymbol{a}_{i}^{k}
$$

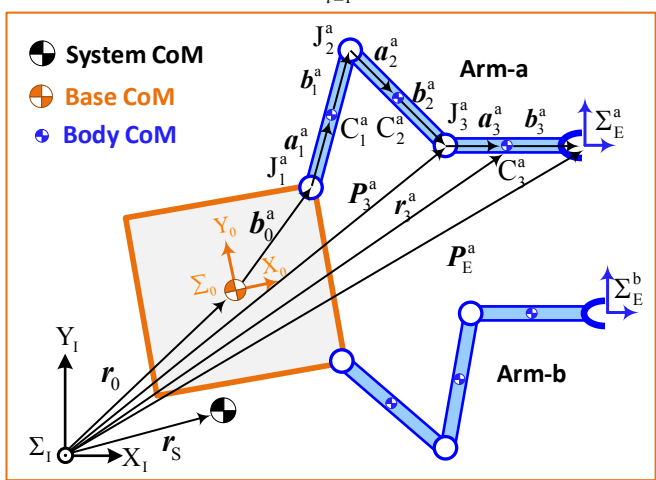

Fig. 6. Diagram of a dual-arm space robot

TABLE I DEFINITIONS OF SYMBOLS AND VARIABLES

\begin{tabular}{ll}
\hline \hline \multicolumn{1}{c}{ Symbols } & Definitions \\
\hline$\Sigma_{\mathrm{I}}, \Sigma_{0}, \Sigma_{\mathrm{T}}, \Sigma_{\mathrm{E}}^{k}$ & Inertial, base, object, and end-effector of arm- $k$ frames \\
$\mathrm{J}_{i}^{k}, \mathrm{C}_{i}^{k}$ & Joint $i$ and center of mass $(\mathrm{CoM})$ of body $i$ of arm- $k$ \\
$m_{i}^{k}, M$ & Masses of body $i$ of arm- $k$ and the whole system \\
$\boldsymbol{r}_{\mathrm{S}}$ & Position vector of the whole system CoM \\
$\boldsymbol{r}_{0}, \boldsymbol{r}_{\mathrm{T}}, \boldsymbol{r}_{i}^{k}$ & Position vectors from $\Sigma_{\mathrm{I}}$ to $\Sigma_{0}, \Sigma_{\mathrm{T}}$, and $\mathrm{C}_{i}^{k}$ \\
$\boldsymbol{p}_{i}^{k}, \boldsymbol{p}_{\mathrm{E}}^{k}$ & Position vectors from $\Sigma_{\mathrm{I}}$ to $\mathrm{J}_{i}^{k}$ and $\Sigma_{\mathrm{E}}^{k}$ \\
$\boldsymbol{a}_{i}^{k}, \boldsymbol{b}_{i}^{k}$ & Position vectors from $\mathrm{J}_{i}^{k}$ to $\mathrm{C}_{i}^{k}$ and from $\mathrm{C}_{i}^{k}$ to $\mathbf{J}_{i+1}^{k}$ \\
$\boldsymbol{l}_{i}^{k}$ & Position vector from $\mathrm{J}_{i}^{k}$ to $\mathbf{J}_{i+1}^{k}$ \\
$\theta_{i}^{k}$ & Angle value of $\mathbf{J}_{i}^{k}$ \\
$\boldsymbol{v}_{0}, \boldsymbol{v}_{i}^{k}, \boldsymbol{v}_{\mathrm{E}}^{k}$ & Linear velocities of $\Sigma_{0}, \mathrm{C}_{i}^{k}$, and $\Sigma_{\mathrm{E}}^{k}$ \\
$\boldsymbol{\omega}_{0}, \boldsymbol{\omega}_{i}^{k}, \boldsymbol{\omega}_{\mathrm{E}}^{k}$ & Angular velocities of $\Sigma_{0}, \mathrm{C}_{i}^{k}$, and $\Sigma_{\mathrm{E}}^{k}$ \\
$\boldsymbol{z}_{i}^{k}$ & Unit vector that specifies the rotational axis of $\mathrm{J}_{i}^{k}$ \\
$\mathbf{E}$ & Unit matrix \\
$\tilde{\boldsymbol{k}}$ & Skew-symmetric matrix of vector $\boldsymbol{k}$ \\
\hline \hline
\end{tabular}

where $n_{k}$ represents the number of bodies in arm- $k$. The velocity of the CoM of body $i$ can be calculated as

$$
\boldsymbol{v}_{i}^{k}=\dot{\boldsymbol{r}}_{i}^{k}=\boldsymbol{v}_{0}+\boldsymbol{\omega}_{0} \times\left(\boldsymbol{r}_{i}^{k}-\boldsymbol{r}_{0}\right)+\sum_{j=1}^{i}\left\{\boldsymbol{z}_{j}^{k} \times\left(\boldsymbol{r}_{i}^{k}-\boldsymbol{P}_{j}^{k}\right)\right\} \dot{\theta}_{j}^{k}
$$

The velocity of the end-effector of arm- $k$ is expressed as

$$
\boldsymbol{v}_{\mathrm{E}}^{k}=\dot{\boldsymbol{P}}_{\mathrm{E}}^{k}=\boldsymbol{v}_{0}+\boldsymbol{\omega}_{0} \times\left(\boldsymbol{P}_{\mathrm{E}}^{k}-\boldsymbol{r}_{0}\right)+\sum_{i=1}^{n_{k}}\left\{\boldsymbol{z}_{i}^{k} \times\left(\boldsymbol{P}_{\mathrm{E}}^{k}-\boldsymbol{P}_{i}^{k}\right)\right\} \dot{\theta}_{i}^{k}
$$

The angular velocity of joint $i$ and the end-effector of arm- $k$ can be deduced as

$$
\boldsymbol{\omega}_{i}^{k}=\boldsymbol{\omega}_{0}+\sum_{j=1}^{i} \boldsymbol{z}_{j}^{k} \dot{\theta}_{j}^{k}, \boldsymbol{\omega}_{\mathrm{E}}^{k}=\boldsymbol{\omega}_{0}+\sum_{i=1}^{n_{k}} z_{i}^{k} \dot{\theta}_{i}^{k}
$$

The free-flying kinematic equation can be derived by combining (5) and (6).

$$
\begin{aligned}
& {\left[\begin{array}{c}
\boldsymbol{v}_{\mathrm{E}}^{k} \\
\boldsymbol{\omega}_{\mathrm{E}}^{k}
\end{array}\right]=\boldsymbol{J}_{\mathrm{B}}^{k}\left[\begin{array}{c}
\boldsymbol{v}_{0} \\
\boldsymbol{\omega}_{0}
\end{array}\right]+\boldsymbol{J}_{\mathrm{M}}^{k} \dot{\boldsymbol{\theta}}^{k}} \\
& \boldsymbol{J}_{\mathrm{B}}^{k}=\left[\begin{array}{cc}
\mathbf{E} & -\tilde{\boldsymbol{P}}_{0 \mathrm{E}}^{k} \\
\mathbf{0} & \mathbf{E}
\end{array}\right]
\end{aligned}
$$

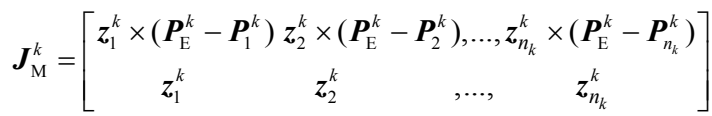

$$
\begin{aligned}
& \boldsymbol{P}_{0 \mathrm{E}}^{k}=\boldsymbol{P}_{\mathrm{E}}^{k}-\boldsymbol{r}_{0}=\boldsymbol{b}_{0}^{k}+\sum_{i=1}^{n_{k}}\left(\boldsymbol{a}_{i}^{k}+\boldsymbol{b}_{i}^{k}\right)
\end{aligned}
$$


where $\theta^{k}$ is the joint angle vector of arm- $k$, $\boldsymbol{\theta}^{k}=\left[\theta_{1}^{k}, \theta_{2}^{k}, \ldots, \theta_{n_{k}}^{k}\right]^{\mathrm{T}} ; \boldsymbol{J}_{\mathrm{B}}^{k}$ represents the Jacobian matrix of the base that corresponds to arm- $k$; and $\boldsymbol{J}_{\mathrm{M}}^{k}$ represents the Jacobian matrix of arm- $k$.

For the free-flying mode, if the base can keep still, namely, $\boldsymbol{v}_{0}=\mathbf{0}$ and $\boldsymbol{\omega}_{0}=\mathbf{0}$, then (7) can be reduced to

$$
\left[\begin{array}{c}
\boldsymbol{v}_{\mathrm{E}}^{k} \\
\boldsymbol{\omega}_{\mathrm{E}}^{k}
\end{array}\right]=\boldsymbol{J}_{\mathrm{M}}^{k} \dot{\boldsymbol{\theta}}^{k}=\left[\begin{array}{c}
\boldsymbol{J}_{\mathrm{Mv}}^{k} \\
\boldsymbol{J}_{\mathrm{M} \omega}^{k}
\end{array}\right] \dot{\boldsymbol{\theta}}^{k}
$$

where $\boldsymbol{J}_{\mathrm{Mv}}^{k}$ represents the sub-Jacobian matrix that corresponds to the linear velocity terms. Equation (11) is equivalent to the kinematic model of the base-fixed robots.

\section{B. Static Force Analysis}

When the end-effector contacts an object, it generates force and torque on the contact point and surface. According to (11), the static force of arm- $k$ can be expressed as

$$
\tau^{k}=J_{\mathrm{Mv}}^{k \mathrm{~T}} \boldsymbol{f}_{\mathrm{E}}^{k}
$$

where $\tau^{k}$ is the joint torque vector of arm- $k$ and $\boldsymbol{\tau}^{k}=\left[\tau_{1}^{k}, \tau_{2}^{k}, \ldots, \tau_{n_{k}}^{k}\right]^{\mathrm{T}} ; \boldsymbol{J}_{\mathrm{Mv}}^{k \mathrm{~T}}$ is the force Jacobian matrix of arm- $k$; and $\boldsymbol{f}_{\mathrm{E}}^{k}$ is the contact force of the end-effector of arm- $k$.

\section{CAGING-PAIR METHOD}

Rodriguez et al. [34] investigated the relationship between caging and grasping and analogously proposed the concepts of pre-grasping and grasping cages. Seo et al. [35] proposed a caging method in which curved-contact-surface effectors are used, which employ a similar strategy of using parallel-jaw effectors to clamp antipodal pairs of convex polyhedral objects. Inspired by [34], [35], we propose a novel caging-pair method that utilizes the dual-arm simulator with hollow-shaped effector pairs to capture non-graspable objects. Subsequently, we will discuss the caging pair principle, the design methodology of the end-effectors, and the concept of caging compatibility.

\section{A. Related Concepts and Principle of Cage Pairs}

As a trade-off method, caging aims at constructing obstacles to confine an object; hence, caging differs from grasping. An intuitive example of caging and grasping is illustrated in Fig. 7. Fig. 7(a) shows a typical caging, in which four point effectors form a closed planar region to constrain a 2D object. By contrast, Fig. 7(b) shows a form-closure grasping, in which the locations of four contact points on the object can completely immobilize the object according to its geometric constraints. Meanwhile, the stable static equilibrium condition can be guaranteed if the contact model is based on the following ideal and conservative assumptions: all contacts are frictionless and rigid and each contact force $f_{i}(i=\{\mathrm{a}, \mathrm{b}, \mathrm{c}, \mathrm{d}\})$ is normal to the surface [34]. In the above example, four point effectors are used; hence, a simple strategy is to use a hollow-shaped effector to replace each pair of point effectors. An example of using a pair of hollow-shaped effectors to cage a $3 \mathrm{D}$ object is presented in Fig. 8. Fig. 8(a) illustrates the pre-grasping cage, in which two circular effectors can squeeze inward (or stretch outward) while the object cannot escape. Fig. 8(b) illustrates the grasping cage, in which two circular effectors move to the limit position of the squeezing motion. Based on the ideal contact, for the grasping cage, the external force and torque that act on the object sum to zero. It should be noted that caging does not absolutely immobilize an object. In some cases, it allows the object to maintain DOFs; for example, the object in Fig. 8(b) can rotate around axis $\xi$. The proposed method is based on the geometric principle that is illustrated in Fig. 8(a), in which $l$ is the distance between the antipodal pair

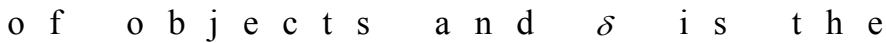

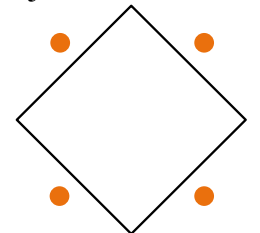

(a) Caging

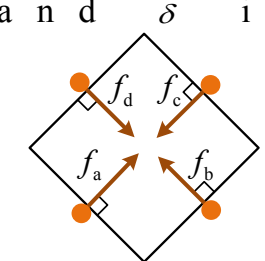

(b) Grasping
Fig. 7. Relationship between caging and grasping

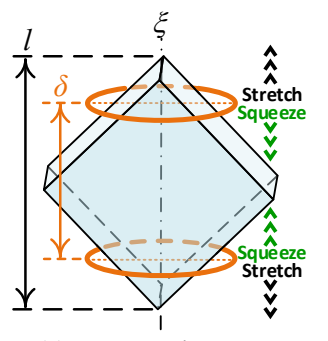

(a) Pre-grasping cage

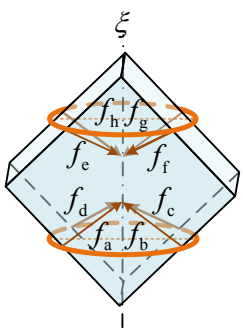

(b) Grasping cage
Fig. 8. Caging by a circular effector pair

distance between the cage pair. Similar to the strategy of using curved-contact-surface effector pairs [35], if condition $\delta<l$ is satisfied, the object can be caged using dual-arm robots. Generally, dual-arm motion strategies can be classified as symmetric or asymmetric [36]. For the symmetric motion, both arms play the same role; the asymmetric motion is a more general manner, in which each arm performs a different role, such as in assembly or machining tasks [37]. In outer space, considering the particularity of caging non-graspable objects, we choose the symmetric capture motion based on two reasons. One reason is due to the physical property of floating objects in space, i.e., if we let one arm touch the floating object first, it may induce the motion or rotation of the object. On the other hand, the proposed method cannot hold objects rigidly, so symmetric capture motions [38] are more suitable for dual-arm space robots to cage objects. In addition, the end-effector is important in our method and a geometric methodology of designing end-effectors will be presented in the following.

\section{B. Methodology for the Design of Caging-Pair End-Effectors}

The antipodal pairs of non-graspable objects include the vertex-vertex, edge-edge, and vertex-face pairs, which are regarded as geometrical features to be caged. A simple methodology from clamping to caging is proposed to design end-effectors. As illustrated in Fig. 9, $\square$ represents a plane and there exist four parallel planes: $\square \mathrm{P}, \square \mathrm{G}, \square \mathrm{C}$, and $\square \mathrm{B}$. In Figs. 9(a1), 9(b1), and 9(c1), $\square \mathrm{P}$ and $\square \mathrm{B}$ can be regarded as two parallel-jaw effectors that form a clamp [39] for capturing an object. Although they appear to realize static equilibrium under the conservative contact model, they may become 
unstable if the parallel-jaw effectors are misaligned slightly. Here, we assume that if the element of the antipodal pairs is a convex hull, then the parallel-jaw planes can be squeezed to penetrate the object, namely, $\square \mathrm{P}$ can be moved to $\square \mathrm{G}$ and $\square \mathrm{B}$ can be moved to $\square \mathrm{C}$. Geometrically, this penetration process involves a process from clamping to caging and the intersecting lines (or the intersecting line and contact face) can cage the objects. As illustrated in Figs. 9(a2), 9(b2), and 9(c2), a series of hollow-shaped end-effectors (including circular, triangular, and rectangular effectors) are designed. Here, the distance between

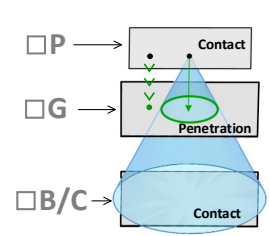

(a1) From clamping to caging

(a) Caging a vertex-face antipodal pair

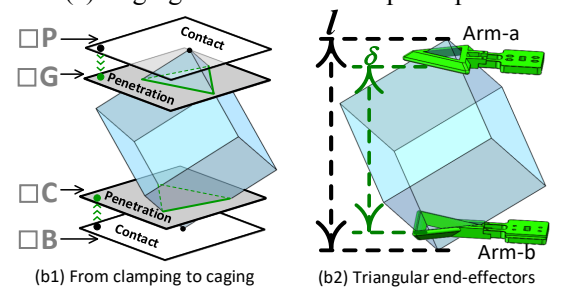

(b) Caging a vertex-vertex antipodal pair

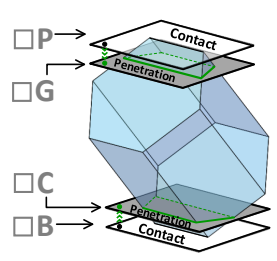

(c1) From clamping to caging

(c) Caging an edge-edge antipodal pair

Fig. 9. Caging via a series of hollow-shaped end-effector pairs

$\square \mathrm{P}$ and $\square \mathrm{B}$ denoted as $d(\mathrm{P}, \mathrm{B})=l$, is the distance between the antipodal pair, and the distance between $\square \mathrm{G}$ and $\square \mathrm{C}$ denoted as $d(\mathrm{G}, \mathrm{C})=\delta$, is the distance between the caging pair. The caging condition is satisfied, namely, $\Delta d=d(\mathrm{P}, \mathrm{B})-d(\mathrm{G}, \mathrm{C})>0$ $\Leftrightarrow \delta<l$; thus, objects are captured by the geometric cage pairs.

\section{Caging Compatibility Evaluation Index}

In this subsection, a caging evaluation index will be introduced. The dual-arm coordinated manipulation is needed in forming a robotic cage, which can usually be categorized into goal-coordinated and bimanual manipulation [40]. For bimanual manipulation, at least one of arms grasps the object rigidly, and the dual-arm robot and the grasped object usually form a closed chain. Also, it should satisfy some constraints, so the relative motion equation between the object and arms can be derived [37]. For caging non-graspable objects, it belongs to goal-coordinated manipulation. As shown in Fig. $8(a)$, the space object is floating in the pre-grasping cage and the end-effector cannot hold the object rigidly, in which the dual-arm robot and the object form an open chain. Thus, it is necessary to plan the joint configuration of pre-grasping cages with desired capturing capability for realizing a stable grasping cage. Yoshikawa [41] proposed the velocity and force manipulability ellipsoids (together with the duality property) to describe the velocity and force transmission characteristics of robotic mechanisms, and used the manipulability ellipsoid volume as a performance index to optimize joint configurations. Chiacchio et al [42] extended velocity and force manipulability ellipsoids to evaluate the performance of dual-arm cooperative robots. Lee [43] proposed a dual-arm manipulability ellipsoid based on the volume of intersection between two individual manipulability ellipsoids. Park and Lee [44] proposed a performance index based on the extended-cooperative-task space representation to optimize joint configurations for dual-arm manipulation. Actually, the above cooperative manipulability indexes [42]-[44] are designed for the closed-chain bimanual manipulation manner. As shown in Fig. 8(b), realizing a grasping cage must exert a squeezing action on the antipodal pairs of objects. Thus, for caging objects, the motion and force need to be considered simultaneously in the assigned directions. Chiu [45] derived the manipulability transmission ratio (MTR) in an assigned direction and proposed the task compatibility index by combining the velocity-MTR (V-MTR) and the force-MTR (F-MTR) with different weight coefficients. Based on Chiu's work, we propose a caging compatibility index for the caging-pair capture, as

$$
\begin{gathered}
C=C\left(\boldsymbol{\theta}^{\mathrm{a}}\right)+C\left(\boldsymbol{\theta}^{\mathrm{b}}\right) \\
C\left(\boldsymbol{\theta}^{k}\right)=\lambda_{\mathrm{v}}^{k}\left(\frac{\alpha\left(\boldsymbol{\theta}^{k}\right)-\alpha_{\min }^{k}}{\alpha_{\max }^{k}-\alpha_{\min }^{k}}\right)+\lambda_{\mathrm{f}}^{k}\left(\frac{\beta\left(\boldsymbol{\theta}^{k}\right)-\beta_{\min }^{k}}{\beta_{\max }^{k}-\beta_{\min }^{k}}\right) \\
\alpha\left(\boldsymbol{\theta}^{k}\right)=\left(\boldsymbol{u}^{k \mathrm{~T}}\left(\boldsymbol{J}_{\mathrm{Mv}}^{k} \boldsymbol{J}_{\mathrm{Mv}}^{k \mathrm{~T}}\right)^{-1} \boldsymbol{u}^{k}\right)^{-1 / 2} \\
\beta\left(\boldsymbol{\theta}^{k}\right)=\left(\boldsymbol{u}^{k \mathrm{~T}}\left(\boldsymbol{J}_{\mathrm{Mv}}^{k} \boldsymbol{J}_{\mathrm{Mv}}^{k \mathrm{~T}}\right) \boldsymbol{u}^{k}\right)^{-1 / 2}
\end{gathered}
$$

where the scalar $C$ is the caging compatibility index for characterizing the dual-arm capture capability along each assigned direction in the current configuration; for arm- $k$, $k=\{\mathrm{a}, \mathrm{b}\} ; C\left(\boldsymbol{\theta}^{k}\right)$ is a normalized index of a single arm, which can eliminate the different orders of magnitude of the V-MTR and F-MTR caused by the arm size; $\boldsymbol{u}^{k}$ is the unit vector of the assigned direction; $\alpha\left(\boldsymbol{\theta}^{k}\right)$ and $\beta\left(\boldsymbol{\theta}^{k}\right)$ are the V-MTR and F-MTR in the direction of $\boldsymbol{u}^{k}$, respectively; $\alpha_{\min }^{k}$ and $\alpha_{\max }^{k}$ are the minimum and maximum values of the V-MTR in joint space, respectively; $\beta_{\min }^{k}$ and $\beta_{\max }^{k}$ are the minimum and maximum values of the F-MTR in joint space, respectively; and $\lambda_{\mathrm{v}}^{k}$ and $\lambda_{\mathrm{f}}^{k}$ are weight coefficients that correspond to the V-MTR and F-MTR, respectively, which satisfy constraints: $0 \leq \lambda_{\mathrm{v}}^{k} \leq 1,0 \leq \lambda_{\mathrm{f}}^{k} \leq 1$, and $\lambda_{\mathrm{v}}^{k}+\lambda_{\mathrm{f}}^{k}=1$. Due to the duality property, we know the velocity and force ellipsoids have the same principal axes but their corresponding dimensions are reciprocal relations. It should be noted that the definition of (14) is not in conflict with the duality property because the proposed convex index is based on the V-MTR and F-MTR. As the MTR describes the manipulability along the assigned direction, which is not same with the principal axis of the manipulability ellipsoid, the duality property does not suit the V-MTR and F-MTR. Meanwhile, the V-MTR and F-MTR are not reciprocal relations and it exists that both V-MTR and F-MTR of Configuration-A are higher than those of Configuration-B, which can be proven with the subsequent analysis results. Next, we will use the caging compatibility index to optimize the joint configuration of the pre-grasping cage, and we will prove that the proposed index is not equivalent to maximizing the ellipsoid volume or a single V/F-MTR. 


\section{CAGING ANALYSIS AND EXPERIMENTS}

\section{A. Configuration Analysis of Pre-Grasping Cages}

For steady communication between the space robot and Earth, the attitude of the base should be stabilized [28]. Thus, in this study, we assume that the base keeps an assigned attitude and the shape and pose information of objects can be measured. However, the relative position relationship between the base and the object is not certain, i.e., the berth position of the base [8] is not determined. Therefore, there may exist many feasible pre-grasping cages for the same object. In Fig.10, for simplicity, we set $\Sigma_{1}$ and $\Sigma_{0}$ have the same orientation; $\boldsymbol{r}_{0}$ represents the berth position; and $\boldsymbol{P}_{0 i}^{k}, \boldsymbol{P}_{0 \mathrm{E}}^{k}$, and $\boldsymbol{P}_{0 \mathrm{~T}}$, represent position vectors from $\Sigma_{0}$ to $\mathrm{J}_{i}^{k}, \Sigma_{\mathrm{E}}^{k}$, and $\Sigma_{\mathrm{T}}$, respectively. Based on the caging compatibility and the relative attitude and distance constraints between the antipodal pair of objects, the $\begin{array}{lllllllllllllllll}\mathrm{p} & \mathrm{r} & \mathrm{o} & \mathrm{b} & \mathrm{l} & \mathrm{e} & \mathrm{m} & \mathrm{o} & \mathrm{f} & \mathrm{p} & \mathrm{l} & \mathrm{a} & \mathrm{n} & \mathrm{n} & \mathrm{i} & \mathrm{n} & \mathrm{g}\end{array}$

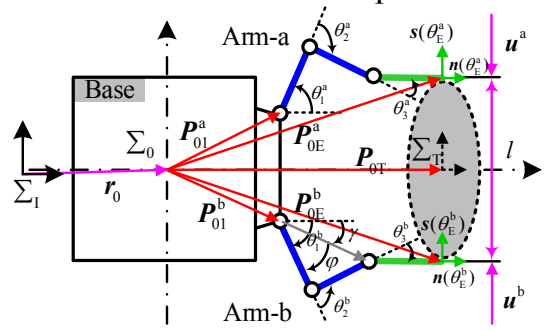

(a) Pre-grasping cage 1

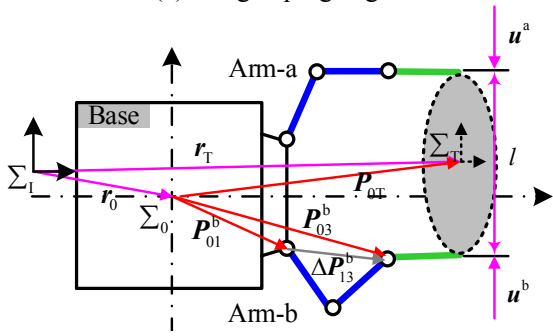

(b) Pre-grasping cage 2

Fig. 10. Two pre-grasping cages

TABLE II GEOMETRICAL PARAMETERS OF FIG. 10(a)

\begin{tabular}{cccc}
\hline \hline Arm- $k$ & Body $i$ & $\left\|\boldsymbol{l}_{i}^{k}\right\|(\mathrm{m})$ & $\theta_{i}^{k}\left({ }^{\circ}\right)$ \\
\hline & 1 & 0.085 & $75 /-75$ \\
$\mathrm{a} / \mathrm{b}$ & 2 & 0.085 & $-90 / 90$ \\
& 3 & 0.085 & $15 /-15$ \\
\hline \hline
\end{tabular}

the pre-grasping configuration $\boldsymbol{\theta}=\left[\boldsymbol{\theta}^{\mathrm{aT}} \boldsymbol{\theta}^{\mathrm{bT}}\right]^{\mathrm{T}}$ for caging objects can be formulated as the following optimization problem:

$$
\begin{aligned}
& \text { Maximize : } C_{\mathrm{d}}=C\left(\boldsymbol{\theta}^{\mathrm{a}}\right)+C\left(\boldsymbol{\theta}^{\mathrm{b}}\right) \\
& \text { Subject to: }\left\{\begin{array}{c}
\boldsymbol{n}\left(\theta_{\mathrm{E}}^{\mathrm{a}}\right)^{\mathrm{T}} \boldsymbol{u}^{\mathrm{a}}=0 \\
\boldsymbol{n}\left(\theta_{\mathrm{E}}^{\mathrm{b}}\right)^{\mathrm{T}} \boldsymbol{u}^{\mathrm{b}}=0 \\
\boldsymbol{P}_{\mathrm{OE}}^{\mathrm{a}}-\boldsymbol{P}_{0 \mathrm{E}}^{\mathrm{b}}=l \cdot \boldsymbol{u}^{\mathrm{a}}
\end{array},\left\{\begin{array}{l}
\boldsymbol{\theta}_{\min }^{\mathrm{a}} \leq \boldsymbol{\theta}^{\mathrm{a}} \leq \boldsymbol{\theta}_{\max }^{\mathrm{a}} \\
\boldsymbol{\theta}_{\min }^{\mathrm{b}} \leq \boldsymbol{\theta}^{\mathrm{b}} \leq \boldsymbol{\theta}_{\max }^{\mathrm{b}}
\end{array}\right.\right.
\end{aligned}
$$

where $C_{\mathrm{d}}$ is the desired caging compatibility index; $l_{i}^{k}$ is the position vector of body $i$ of arm- $k(k=\{\mathrm{a}, \mathrm{b}\}), \boldsymbol{l}_{i}^{k}=\boldsymbol{a}_{i}^{k}+\boldsymbol{b}_{i}^{k} ; \theta_{\mathrm{E}}^{k}$ is the attitude angle of the end-effector of arm- $k ; \boldsymbol{n}\left(\theta_{\mathrm{E}}^{k}\right)$ and $\boldsymbol{s}\left(\theta_{\mathrm{E}}^{k}\right)$ are the unit frame vectors of $\Sigma_{\mathrm{E}}^{k} ; \boldsymbol{u}^{k}$ is the pre-grasping direction of arm- $k$; and $\boldsymbol{\theta}_{\min }^{k}$ and $\boldsymbol{\theta}_{\max }^{k}$ are the minimum and maximum joint vector values, respectively, of arm- $k$. For this capture task, if $\boldsymbol{\theta}$ is determined, we can obtain $\boldsymbol{P}_{0 \mathrm{~T}}, \boldsymbol{r}_{0}, \boldsymbol{P}_{\mathrm{E}}^{k}$, and $\theta_{\mathrm{E}}^{k}$. The detailed calculation and derivation are given below.

For the proposed simulator, there are six undetermined variables in the configuration space for planning the optimal pre-grasping configuration. Equation (17) presents the position and attitude constraints of capturing the object, which include four equality constraints. Thus, there are only two free variables in this problem. In this study, $\theta_{1}^{a}$ and $\theta_{2}^{a}$ are selected as free variables, and other joint variables, namely, $\theta_{3}^{\mathrm{a}}, \theta_{1}^{\mathrm{b}}, \theta_{2}^{\mathrm{b}}$, and $\theta_{3}^{\mathrm{b}}$, can be calculated according to the following geometrical relationships in Fig. 10 and the symbol definitions in Table I.

$$
\begin{gathered}
\theta_{3}^{\mathrm{a}}=\theta_{\mathrm{E}}^{\mathrm{a}}-\theta_{1}^{\mathrm{a}}-\theta_{2}^{\mathrm{a}} \\
\boldsymbol{r}_{0}=\boldsymbol{r}_{\mathrm{T}}-\boldsymbol{P}_{0 \mathrm{~T}} \\
\boldsymbol{P}_{\mathrm{E}}^{k}=\boldsymbol{r}_{0}+\boldsymbol{P}_{0 \mathrm{E}}^{k} \\
\boldsymbol{P}_{0 \mathrm{~T}}=\boldsymbol{P}_{0 \mathrm{E}}^{\mathrm{a}}+0.5 \cdot l \cdot \boldsymbol{u}^{\mathrm{a}} \\
\boldsymbol{P}_{0 \mathrm{E}}^{k}=\boldsymbol{P}_{01}^{k}+\left\|\boldsymbol{l}_{1}^{k}\right\|\left[\begin{array}{cc}
\mathrm{c}_{1}^{k} & \mathrm{~s}_{1}^{k}
\end{array}\right]^{\mathrm{T}}+\left\|\boldsymbol{l}_{2}^{k}\right\|\left[\begin{array}{ll}
\mathrm{c}_{12}^{k} & \mathrm{~s}_{12}^{k}
\end{array}\right]^{\mathrm{T}}+\left\|\boldsymbol{l}_{3}^{k}\right\|\left[\begin{array}{ll}
\mathrm{c}_{123}^{k} & \mathrm{~s}_{123}^{k}
\end{array}\right]^{\mathrm{T}} \\
\boldsymbol{P}_{03}^{\mathrm{a}}=\boldsymbol{P}_{0 \mathrm{E}}^{\mathrm{a}}-\left\|\boldsymbol{l}_{3}^{\mathrm{a}}\right\|\left[\begin{array}{ll}
\mathrm{c}_{123}^{\mathrm{a}} & \mathrm{s}_{123}^{\mathrm{a}}
\end{array}\right]^{\mathrm{T}} \\
\boldsymbol{P}_{0 \mathrm{E}}^{\mathrm{b}}=\boldsymbol{P}_{0 \mathrm{E}}^{\mathrm{a}}+l \cdot \boldsymbol{u}^{\mathrm{a}} \\
\boldsymbol{P}_{03}^{\mathrm{b}}=\boldsymbol{P}_{03}^{\mathrm{a}}+l \cdot \boldsymbol{u}^{\mathrm{a}} \\
\Delta \boldsymbol{P}_{13}^{\mathrm{b}}=\boldsymbol{P}_{03}^{\mathrm{b}}-\boldsymbol{P}_{01}^{\mathrm{b}} \\
\theta_{1}^{\mathrm{b}}=\gamma+\varphi
\end{gathered}
$$

where $\mathrm{c}_{1}^{k}=\cos \left(\theta_{1}^{k}\right), \mathrm{c}_{12}^{k}=\cos \left(\theta_{1}^{k}+\theta_{2}^{k}\right), \mathrm{c}_{123}^{k}=\cos \left(\theta_{1}^{k}+\theta_{2}^{k}+\theta_{3}^{k}\right)$, $s_{1}^{k}=\sin \left(\theta_{1}^{k}\right), s_{12}^{k}=\sin \left(\theta_{1}^{k}+\theta_{2}^{k}\right)$, and $s_{123}^{k}=\sin \left(\theta_{1}^{k}+\theta_{2}^{k}+\theta_{3}^{k}\right)$.

Furthermore, the following equations are obtained:

$$
\begin{gathered}
\cos \left(\theta_{2}^{\mathrm{b}}\right)=\frac{\left\|\Delta \boldsymbol{P}_{13}^{\mathrm{b}}\right\|^{2}-\left\|\boldsymbol{l}_{1}^{\mathrm{b}}\right\|^{2}-\left\|\boldsymbol{I}_{2}^{\mathrm{b}}\right\|^{2}}{2 \cdot\left\|\boldsymbol{l}_{1}^{\mathrm{b}}\right\| \cdot\left\|\boldsymbol{l}_{2}^{\mathrm{b}}\right\|} \\
\theta_{2}^{\mathrm{b}}(1)=\left|\theta_{2}^{\mathrm{b}}\right|, \theta_{2}^{\mathrm{b}}(2)=-\left|\theta_{2}^{\mathrm{b}}\right|
\end{gathered}
$$

where $\theta_{2}^{\mathrm{b}}$ may have two solutions, which are denoted as $\theta_{2}^{\mathrm{b}}(1)$ and $\theta_{2}^{\mathrm{b}}(2)$, and $\Delta \boldsymbol{P}_{13}^{\mathrm{b}}=\left[\Delta P_{13 \mathrm{x}}^{\mathrm{b}}, \Delta P_{13 \mathrm{y}}^{\mathrm{b}}\right]^{\mathrm{T}}$. As shown in Fig. 10(a),

$$
\gamma=\operatorname{Atan} 2\left(\Delta P_{13 \mathrm{y}}^{\mathrm{b}}, \Delta P_{13 \mathrm{x}}^{\mathrm{b}}\right)
$$

Similarly, angle $\varphi$ can be calculated via

$$
\cos (\varphi)=\frac{\left\|\Delta \boldsymbol{P}_{13}^{\mathrm{b}}\right\|^{2}+\left\|\boldsymbol{l}_{1}^{\mathrm{b}}\right\|^{2}-\left\|\boldsymbol{l}_{2}^{\mathrm{b}}\right\|^{2}}{2 \cdot\left\|\boldsymbol{l}_{1}^{\mathrm{b}}\right\| \cdot\left\|\Delta \Delta \boldsymbol{P}_{13}^{\mathrm{b}}\right\|}
$$

Furthermore, we can deduce

$$
\begin{array}{r}
\theta_{1}^{\mathrm{b}}=\left\{\begin{array}{l}
\gamma-|\varphi|, \theta_{2}^{\mathrm{b}}>0 \\
\gamma+|\varphi|, \theta_{2}^{\mathrm{b}}<0
\end{array}\right. \\
\theta_{3}^{\mathrm{b}}=\theta_{\mathrm{E}}^{\mathrm{b}}-\theta_{1}^{\mathrm{b}}-\theta_{2}^{\mathrm{b}}
\end{array}
$$

where $\theta_{1}^{\mathrm{b}}$ and $\theta_{3}^{\mathrm{b}}$ have two solutions, similar to $\theta_{2}^{\mathrm{b}}$.

According to the preceding analysis, only two free variables need to be determined in this problem. In addition, for the numerical computation, it is not necessary to obtain the absolute optimal pre-grasping configuration. Typically, the near-optimal pre-grasping configurations are suitable for the capture task. Based on the Monte Carlo method, an algorithm of planning the near-optimal pre-grasping configurations via optimizing the caging compatibility index is introduced as follows.

\footnotetext{
Algorithm 1: Planning of the near-optimal pre-grasping configuration

Input: $\boldsymbol{\theta}_{\min }^{k}, \boldsymbol{\theta}_{\max }^{k}, \lambda_{\mathrm{v}}^{k}, \lambda_{\mathrm{f}}^{k}, \alpha_{\min }^{k}, \alpha_{\max }^{k}, \beta_{\min }^{k}, \beta_{\max }^{k},\left\|\boldsymbol{l}_{i}^{k}\right\|, \boldsymbol{n}\left(\theta_{\mathrm{E}}^{k}\right), \boldsymbol{u}^{k}$, $\theta_{\mathrm{E}}^{k}, l, k=\{\mathrm{a}, \mathrm{b}\}$.

Output: $\theta_{\mathrm{NO}}$

1 Initialization

(a) Generate the workspace point cloud $\boldsymbol{P}_{0 \mathrm{E}}^{k}$ via (22).

(b) Generate the workspace boundary regions $\boldsymbol{D}^{k}$.

(c) Initialize a null set, namely, $\boldsymbol{X}=[$ ] , for storing data.

2 Determine the near-optimal configuration.
} 
(a) Generate the joint candidates, namely, $\theta_{1(s)}^{\mathrm{a}}$ and $\theta_{2(s)}^{\mathrm{a}}$, based on Monte Carlo sampling, where $s$ is the sth sampling and $s=1,2, \ldots, n_{\mathrm{s}}$.

(b) For $s=1,2, \ldots, n$, solve for $\theta_{3(s)}^{\mathrm{a}}$ using (18).

If $\theta_{3(s)}^{\mathrm{a}} \in\left[\theta_{3 \min }^{\mathrm{a}} \theta_{3 \max }^{\mathrm{a}}\right]$, then solve for $\boldsymbol{P}_{0 \mathrm{E}(s)}^{\mathrm{b}}$ using (24).

If $\boldsymbol{P}_{0 \mathrm{E}(s)}^{\mathrm{b}} \in \boldsymbol{D}^{\mathrm{b}}$, then solve for $\theta_{2(s)}^{\mathrm{b}}(1)$ and $\theta_{2(s)}^{\mathrm{b}}(2)$ using (29),

solve for $\theta_{1(s)}^{\mathrm{b}}(1)$ and $\theta_{1(s)}^{\mathrm{b}}(2)$ using (32), and solve for $\theta_{3(s)}^{\mathrm{b}}(1)$

and $\theta_{3(s)}^{\mathrm{b}}(2)$ using (33).

If $\boldsymbol{\theta}_{(s)}^{\mathrm{b}}(1) \in\left[\boldsymbol{\theta}_{\min }^{\mathrm{b}} \boldsymbol{\theta}_{\max }^{\mathrm{b}}\right]$, then solve for $C_{\mathrm{d}(s)}(1)$ using

$(13-16)$; save $\left[\boldsymbol{\theta}_{(s)}^{\mathrm{aT}}, \boldsymbol{\theta}_{(s)}^{\mathrm{bT}}(1), C_{\mathrm{d}(s)}(1)\right]^{\mathrm{T}}$ in $\boldsymbol{X}$.

End If.

If $\boldsymbol{\theta}_{(\mathrm{s})}^{\mathrm{b}}(2) \in\left[\boldsymbol{\theta}_{\min }^{\mathrm{b}} \boldsymbol{\theta}_{\max }^{\mathrm{b}}\right]$, then solve for $C_{\mathrm{d}(s)}(2)$ using

$(13-16)$; save $\left[\boldsymbol{\theta}_{(s)}^{\mathrm{aT}}, \boldsymbol{\theta}_{(s)}^{\mathrm{bT}}(2), C_{\mathrm{d}(s)}(2)\right]^{\mathrm{T}}$ in $\boldsymbol{X}$.

End If.

End If.

End If.

\section{End For.}

(c) Select the near-optimal pre-grasping configuration by setting $\boldsymbol{\theta}_{\mathrm{NO}}=\arg \max \left\{C_{\mathrm{d}}(\boldsymbol{\theta})\right\}$.

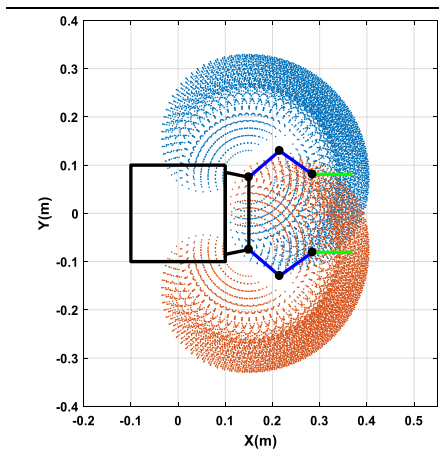

(a) Workspace point cloud

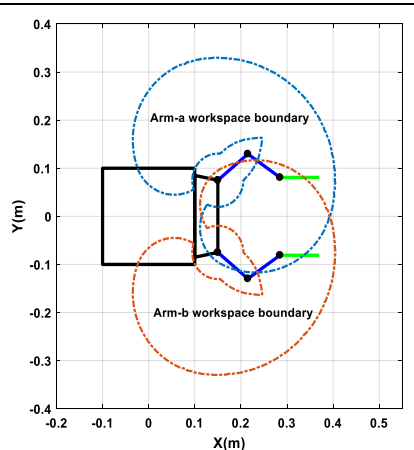

(b) Workspace boundary

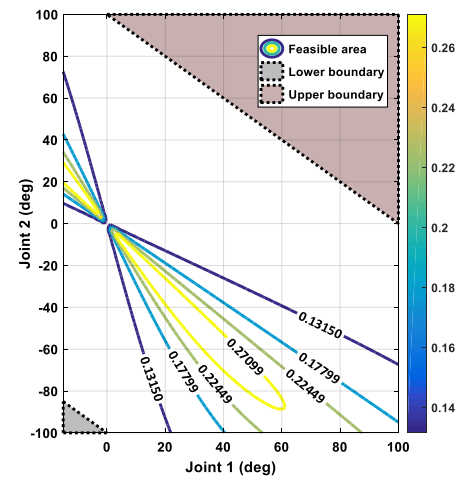

(a) V-MTR of arm-a

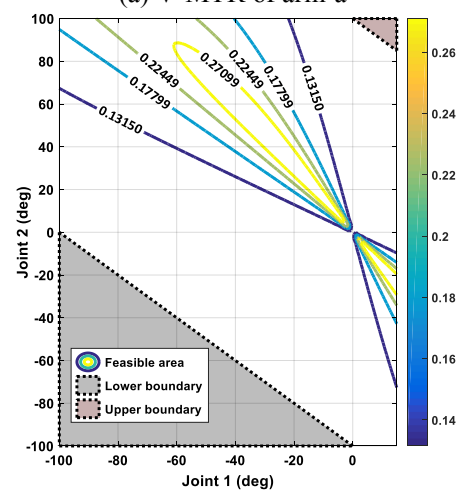

(c) V-MTR of arm-b

Fig. 12. MTR distributions of the proposed simulator

In Algorithm 1, Procedure 1(b) can be achieved by the function boundary in MATLAB, which returns the boundaries of a set of points in 2D/3D space. For the proposed dual-arm space robot simulator, the ranges of the joint angle vectors are set as $\theta_{\min }^{\mathrm{a}}=[-15,-100,-100]^{\mathrm{T}}\left({ }^{\circ}\right), \quad \theta_{\max }^{\mathrm{a}}=[100,100,100]^{\mathrm{T}}\left({ }^{\circ}\right)$, $\boldsymbol{\theta}_{\min }^{\mathrm{b}}=[-100,-100,-100]^{\mathrm{T}}\left({ }^{\circ}\right)$, and $\boldsymbol{\theta}_{\max }^{\mathrm{b}}=[15,100,100]^{\mathrm{T}}\left({ }^{\circ}\right)$; the first joint position vectors of arm-a and arm-b are set as $\boldsymbol{P}_{01}^{\mathrm{a}}=[0.15,0.075]^{\mathrm{T}}$ and $\boldsymbol{P}_{01}^{\mathrm{b}}=[0.15,-0.075]^{\mathrm{T}}$, respectively; and the pre-grasping directions of arm-a and arm-b are set as $\boldsymbol{u}^{\mathrm{a}}=[0,-1]^{\mathrm{T}}$ and $\boldsymbol{u}^{\mathrm{b}}=[0,1]^{\mathrm{T}}$, respectively. Via (17), we can calculate the attitude angles of the end-effectors of arm-a and arm-b, namely, $\theta_{\mathrm{E}}^{\mathrm{a}}=0^{\circ}$ and $\theta_{\mathrm{E}}^{\mathrm{b}}=0^{\circ}$. The workspace point cloud and boundary can be obtained according to Table II, as illustrated in Fig. 11. The V-MTR and F-MTR distribution contours of arm-a and arm-b can be obtained according to (15) and (16), as illustrated in Fig. 12. Here, when $\theta_{\mathrm{E}}^{k}$ is known, we can calculate $\theta_{3}^{k}$ according to the condition $\theta_{3}^{k}=\theta_{\mathrm{E}}^{k}-\theta_{1}^{k}-\theta_{2}^{k}$. Thus, if the ranges of $\theta_{1}^{k}$ and $\theta_{2}^{k}$ are specified, the corresponding MTR of arm- $k$ can be calculated. Meanwhile, the lower and upper boundaries in the configuration space can be determined from the joint angle range of $\theta_{3}^{k}$ and herein only the feasible area in the configuration space is available for determining the joint configuration, as illustrated in Fig. 12. Next, the proposed algorithm will be applied to optimize the near-optimal pre-grasping configuration in the capture analysis and experimental tests.

\section{B. Simulation Studies}

Five simulation studies on caging non-graspable objects are conducted in this subsection. As illustrated in Fig. 9, three objects are captured by three geometric cage pairs. The rectangular cage pair will be used to capture the non-graspable object in Fig. 1(b4) and the circular cage pair will be used to capture the non-graspable object in Fig. 1(b5). Two types of desired caging compatibility are defined according to the symmetry of objects and the concept of caging pairs. The first is the caging pair with same MTRs (such as in Figs. 9(b) and $9(\mathrm{c}))$. We observe the process from the pre-grasping cage to the grasping cage, in which $\square \mathrm{P}$ moves to $\square \mathrm{G}$ and $\square \mathrm{B}$ moves to $\square \mathrm{C}$ over the same distance. Thus, when we plan a pre-grasping cage configuration, arm-a and arm-b should have 
the same V-MTR and F-MTR, namely, $\lambda_{\mathrm{v}}^{\mathrm{a}}=\lambda_{\mathrm{v}}^{\mathrm{b}}$ and $\lambda_{\mathrm{f}}^{\mathrm{a}}=\lambda_{\mathrm{f}}^{\mathrm{b}}$. For the second one, namely, the caging pair with different MTRs(such as Fig. 9(a)), $\square \mathrm{P}$ moves a long distance to $\square \mathrm{G}$ and $\square \mathrm{B}$ moves a short distance to $\square \mathrm{C}$; hence, the weight coefficients are different, namely, $\lambda_{\mathrm{v}}^{\mathrm{a}} \neq \lambda_{\mathrm{v}}^{\mathrm{b}}$ and $\lambda_{\mathrm{f}}^{\mathrm{a}} \neq \lambda_{\mathrm{f}}^{\mathrm{b}}$. Thus, different caging compatibility will be used to optimize the pregrasping configuration. For caging the non-graspable objects (Figs. 1(b1), 1(b2), and 1(b4)), the desired weight coefficients are set as $\lambda_{\mathrm{v}}^{\mathrm{a}}=\lambda_{\mathrm{v}}^{\mathrm{b}}=0.4$ and $\lambda_{\mathrm{f}}^{\mathrm{a}}=\lambda_{\mathrm{f}}^{\mathrm{b}}=0.6$; hence, arm-a and arm-b should have high F-MTR. For caging the non-graspable object (Fig. 1(b3)), the desired weight coefficients are set as $\lambda_{\mathrm{v}}^{\mathrm{a}}=0.75, \lambda_{\mathrm{f}}^{\mathrm{a}}=0.25, \lambda_{\mathrm{v}}^{\mathrm{b}}=0.1$, and $\lambda_{\mathrm{f}}^{\mathrm{b}}=0.9$. For arm-a, the weight coefficient of its V-MTR, namely, $\lambda_{v}^{a}$, should dominate the weight coefficient of its F-MTR, namely, $\lambda_{\mathrm{f}}^{\mathrm{a}}$; for arm-b, the weight coefficient of its F-MTR, namely, $\lambda_{\mathrm{f}}^{\mathrm{b}}$, should dominate the weight coefficient of its V-MTR, namely, $\lambda_{v}^{b}$. For caging the non-graspable object (Fig. 1(b5)), the desired weight coefficients are set as $\lambda_{\mathrm{v}}^{\mathrm{a}}=\lambda_{\mathrm{v}}^{\mathrm{b}}=0.5$ and $\lambda_{\mathrm{f}}^{\mathrm{a}}=\lambda_{\mathrm{f}}^{\mathrm{b}}=0.5$; hence, the V-MTR and

TABLE III ANALYSIS RESULTS OF PLANNING THE

\begin{tabular}{|c|c|c|c|c|c|c|c|c|c|}
\hline \multirow{2}{*}{$\begin{array}{l}\text { Object } \\
l(\mathrm{~m})\end{array}$} & \multirow{2}{*}{$\begin{array}{c}\begin{array}{c}\text { Sampling } \\
\text { points }\end{array} \\
n_{\mathrm{S}} \\
\end{array}$} & \multicolumn{2}{|c|}{ Near-optimal pre-grasping configuration } & \multirow{2}{*}{$\begin{array}{c}\text { Relative position } \\
\boldsymbol{P}_{0 \mathrm{~T}}(\mathrm{~m}) \\
\end{array}$} & \multirow{2}{*}{$\begin{array}{l}\begin{array}{l}\text { V-MTR } \\
\text { of arm-a }\end{array} \\
\alpha\left(\theta^{\mathrm{a}}\right) \\
\end{array}$} & \multirow{2}{*}{$\begin{array}{c}\begin{array}{c}\text { F-MTR } \\
\text { of } \\
\text { arm-a }\end{array} \\
\beta\left(\boldsymbol{\theta}^{\mathrm{a}}\right) \\
\end{array}$} & \multirow{2}{*}{$\begin{array}{l}\begin{array}{c}\text { V-MTR } \\
\text { of } \\
\text { arm-b }\end{array} \\
\alpha\left(\boldsymbol{\theta}^{\mathrm{b}}\right) \\
\end{array}$} & \multirow{2}{*}{$\begin{array}{c}\begin{array}{c}\text { F-MTR } \\
\text { of } \\
\text { arm-b }\end{array} \\
\beta\left(\boldsymbol{\theta}^{\mathrm{b}}\right) \\
\end{array}$} & \multirow{2}{*}{$\begin{array}{c}\begin{array}{c}\text { Caging } \\
\text { compatibility }\end{array} \\
C(\boldsymbol{\theta})\end{array}$} \\
\hline & & $\theta_{\mathrm{NO}}^{\mathrm{a}}\left({ }^{\circ}\right)$ & $\boldsymbol{\theta}_{\mathrm{NO}}^{\mathrm{b}}\left({ }^{\circ}\right)$ & & & & & & \\
\hline \multirow{4}{*}{$\begin{array}{c}\text { Fig. } \\
\begin{array}{c}1(\mathrm{~b} 1) \\
l=0.217\end{array}\end{array}$} & 500 & {$[57.04,-85.86,28.82]^{\mathrm{T}}$} & {$[-58.98,84.18,-25.20]^{\mathrm{T}}$} & {$[0.3557,-0.0032]^{\mathrm{T}}$} & 0.2738 & 3.6521 & 0.2733 & 3.6332 & 0.7182 \\
\hline & 1000 & {$[51.07,-72.05,20.98]^{\mathrm{T}}$} & {$[-49.90,73.27,-23.37]^{\mathrm{T}}$} & {$[0.3678,-0.0022]^{\mathrm{T}}$} & 0.2796 & 3.4993 & 0.2836 & 3.5087 & 0.7266 \\
\hline & 2000 & {$[49.19,-70.54,21.35]^{\mathrm{T}}$} & {$[-49.25,70.48,-21.24]^{\mathrm{T}}$} & {$[0.3697,-0.0001]^{\mathrm{T}}$} & 0.2828 & 3.4826 & 0.2826 & 3.4822 & 0.7274 \\
\hline & 4000 & {$[50.95,-72.89,21.94]^{\mathrm{T}}$} & {$[-50.55,73.31,-22.76]^{\mathrm{T}}$} & {$[0.3674,-0.0008]^{\mathrm{T}}$} & 0.2811 & 3.5065 & 0.2824 & 3.5098 & 0.7277 \\
\hline \multirow{4}{*}{$\begin{array}{c}\text { Fig. } \\
\begin{array}{c}1(\mathrm{~b} 2) \\
l=0.195\end{array}\end{array}$} & 500 & {$[40.27,-61.34,21.07]^{\mathrm{T}}$} & {$[-39.19,62.11,-22.92]^{\mathrm{T}}$} & {$[0.3792,-0.0019]^{\mathrm{T}}$} & 0.2942 & 3.3953 & 0.2934 & 3.4019 & 0.7539 \\
\hline & 1000 & {$[34.43,-51.22,16.79]^{\mathrm{T}}$} & {$[-33.92,51.68,-17.76]^{\mathrm{T}}$} & {$[0.3865,-0.0010]^{\mathrm{T}}$} & 0.2983 & 3.3181 & 0.3003 & 3.3207 & 0.7618 \\
\hline & 2000 & {$[32.22,-48.21,15.99]^{\mathrm{T}}$} & {$[-32.51,47.92,-15.41]^{\mathrm{T}}$} & {$[0.3886,-0.0006]^{\mathrm{T}}$} & 0.3004 & 3.2976 & 0.2985 & 3.2962 & 0.7592 \\
\hline & 4000 & {$[32.67,-48.99,16.32]^{\mathrm{T}}$} & {$[-32.92,48.76,-15.84]^{\mathrm{T}}$} & {$[0.3881,-0.0005]^{\mathrm{T}}$} & 0.3003 & 3.3027 & 0.2989 & 3.3015 & 0.7604 \\
\hline \multirow{4}{*}{$\begin{array}{c}\text { Fig. } \\
1(\mathrm{~b} 3) \\
l=0.150\end{array}$} & 500 & {$[34.99,-55.99,21.00]^{\mathrm{T}}$} & {$[-21.00,55.99,-34.99]^{\mathrm{T}}$} & {$[0.3840,-0.0183]^{\mathrm{T}}$} & 0.2976 & 3.3522 & 0.1855 & 3.4122 & 0.7631 \\
\hline & 1000 & {$[-6.91,12.03,-5.12]^{\mathrm{T}}$} & {$[-6.91,12.03,-5.12]^{\mathrm{T}}$} & {$[0.4040,-0.0026]^{\mathrm{T}}$} & 0.3077 & 3.1537 & 0.3077 & 3.1537 & 0.8155 \\
\hline & 2000 & {$[6.44,-10.32,3.88]^{\mathrm{T}}$} & {$[6.44,-10.32,3.88]^{\mathrm{T}}$} & {$[0.4043,-0.0038]^{\mathrm{T}}$} & 0.3173 & 3.1511 & 0.3173 & 3.1511 & 0.8502 \\
\hline & 4000 & {$[4.77,-7.84,3.07]^{\mathrm{T}}$} & {$[4.77,-7.84,3.07]^{\mathrm{T}}$} & {$[0.4046,-0.0025]^{\mathrm{T}}$} & 0.3166 & 3.1482 & 0.3166 & 3.1482 & 0.8473 \\
\hline \multirow{4}{*}{$\begin{array}{c}\text { Fig. } \\
\text { 1(b4) } \\
l=0.210\end{array}$} & 500 & {$[46.05,-69.13,23.08]^{\mathrm{T}}$} & {$[-47.20,68.04,-20.84]^{\mathrm{T}}$} & {$[0.3722,-0.0021]^{\mathrm{T}}$} & 0.2880 & 3.4663 & 0.2850 & 3.4579 & 0.7376 \\
\hline & 1000 & {$[43.19,-64.95,21.76]^{\mathrm{T}}$} & {$[-44.90,63.18,-18.28]^{\mathrm{T}}$} & {$[0.3759,-0.0033]^{\mathrm{T}}$} & 0.2910 & 3.4272 & 0.2836 & 3.4155 & 0.7347 \\
\hline & 2000 & {$[46.14,-67.43,21.29]^{\mathrm{T}}$} & {$[-45.91,67.65,-21.74]^{\mathrm{T}}$} & {$[0.3731,-0.0004]^{\mathrm{T}}$} & 0.2870 & 3.4512 & 0.2877 & 3.4528 & 0.7392 \\
\hline & 4000 & {$[47.32,-69.99,22.67]^{\mathrm{T}}$} & {$[-47.47,69.85,-22.38]^{\mathrm{T}}$} & {$[0.3711,-0.0003]^{\mathrm{T}}$} & 0.2864 & 3.4752 & 0.2861 & 3.4741 & 0.7386 \\
\hline \multirow{4}{*}{$\begin{array}{c}\text { Fig. } \\
\begin{array}{c}\text { 1(b5) } \\
l=0.200\end{array}\end{array}$} & 500 & {$[44.73,-70.33,25.60]^{\mathrm{T}}$} & {$[-45.87,69.52,-23.65]^{\mathrm{T}}$} & {$[0.3720,-0.0019]^{\mathrm{T}}$} & 0.2869 & 3.4782 & 0.2880 & 3.4700 & 0.9091 \\
\hline & 1000 & {$[37.81,-55.51,17.70]^{\mathrm{T}}$} & {$[-37.16,56.11,-18.95]^{\mathrm{T}}$} & {$[0.3831,-0.0013]^{\mathrm{T}}$} & 0.2944 & 3.3497 & 0.2970 & 3.3533 & 0.9303 \\
\hline & 2000 & {$[38.95,-58.20,19.25]^{\mathrm{T}}$} & {$[-38.73,58.40,-19.67]^{\mathrm{T}}$} & {$[0.3814,-0.0004]^{\mathrm{T}}$} & 0.2949 & 3.3700 & 0.2955 & 3.3713 & 0.9304 \\
\hline & 4000 & {$[38.32,-56.94,18.62]^{\mathrm{T}}$} & {$[-38.02,57.20,-19.18]^{\mathrm{T}}$} & {$[0.3822,-0.0006]^{\mathrm{T}}$} & 0.2951 & 3.3602 & 0.2961 & 3.3618 & 0.9310 \\
\hline
\end{tabular}

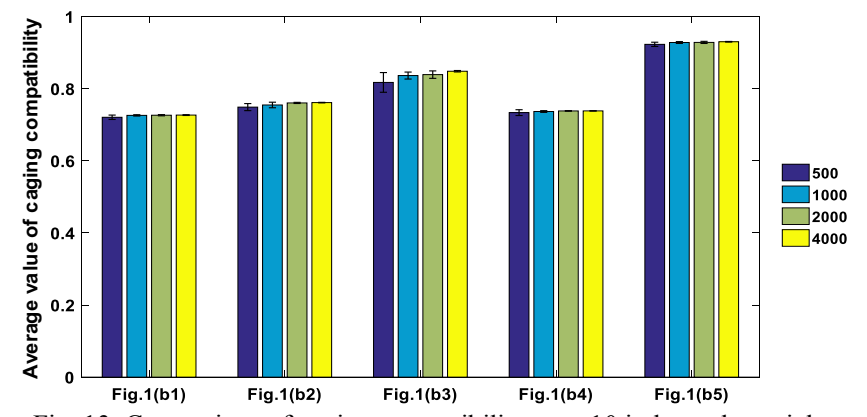

Fig. 13. Comparison of caging compatibility over 10 independent trials

increasing the number of sampling points does not always guarantee an improved simulation result. Moreover, we conduct ten independent trials with four sampling numbers and the average values of the caging compatibility are listed in Fig. 13. The results demonstrate that our algorithm is efficient and the near-optimal solutions can be obtained when the number of sampling points is equal to or larger than 500 .
F-MTR for the two arms are deemed equally important. Here, for the simulation studies, the V-MTR and F-MTR boundaries can be obtained directly from Fig. 12, where $\alpha_{\min }^{\mathrm{a}}=\alpha_{\min }^{\mathrm{b}}=0.085, \alpha_{\max }^{\mathrm{a}}=\alpha_{\max }^{\mathrm{b}}=0.31748, \beta_{\text {min }}^{\mathrm{a}}=\beta_{\min }^{\mathrm{b}}=3.14437$, and $\beta_{\text {max }}^{\mathrm{a}}=\beta_{\text {max }}^{\mathrm{b}}=11.76433$.

According to Algorithm 1, the near-optimal pre-grasping configurations can be obtained. Also, it is necessary to determine a suitable sampling number for the proposed algorithm. Here, the number of sampling points of the Monte Carlo algorithm, namely, $n_{\mathrm{S}}$, is set as 500, 1000, 2000, and 4000 , respectively, and the analysis results are listed in Table III. The caging compatibility indexes are nearly equal in each group. Meanwhile, all the results in Table III have been verified using the MATLAB Robotics Toolbox [46]. In the second group, the simulation with 1000 sampling points outperforms that with 2000 sampling points. The main reason is that the Monte Carlo simulation is a random experiment.

$\begin{array}{llllllllll}\mathrm{T} & \mathrm{h} & \mathrm{e} & \mathrm{r} & \mathrm{e} & \mathrm{f} & \mathrm{o} & \mathrm{r} & \mathrm{e}\end{array}$

Meanwhile, as we mentioned before, V-MTR and F-MTR are not reciprocal relations, such as the V-MTR and F-MTR of arm-b in the third group with 500 sampling points, where $\alpha\left(\boldsymbol{\theta}^{\mathrm{b}}\right) \times \beta\left(\boldsymbol{\theta}^{\mathrm{b}}\right)=0.6330$. It exists that both V-MTR and F-MTR of Configuration-A are higher than those of Configuration-B. For example, in the second group, both V-MTR and F-MTR of arm-b configuration with 4000 sampling points are higher than those with 2000 sampling points. Meanwhile, using the caging compatibility index is different from using a single V-MTR or F-MTR index. For example, in the first group, the sum of V-MTRs of both arms with 2000 sampling points is higher than that with 4000 sampling points, but the analysis results are opposite.

\section{Experimental Verification}

The aim of the experiment tests is to evaluate the robustness and performance of our caging-pair method. As shown in Fig. 14, three types of hollow-shaped end-effector pairs are 
prepared for five non-graspable objects. For each test, the initial configuration of the robotic arms is set as $\boldsymbol{\theta}^{\mathrm{a}}=[90,-90$, $0.0]^{\mathrm{T}}\left({ }^{\circ}\right)$ and $\boldsymbol{\theta}^{\mathrm{b}}=[-90,90,0.0]^{\mathrm{T}}\left({ }^{\circ}\right)$. The analysis results in

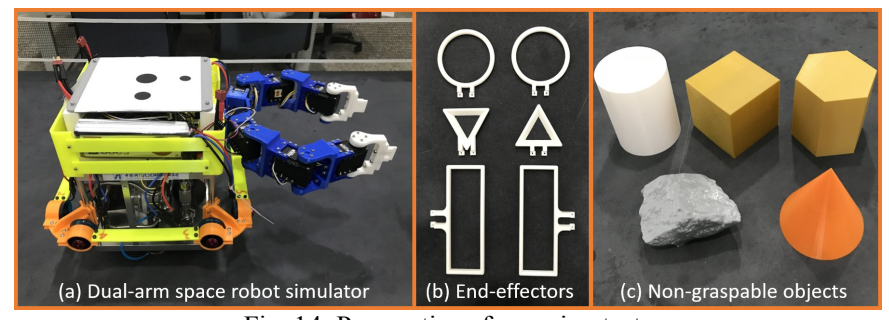

Fig. 14. Preparations for caging tests

Table III with 500 sampling points are used to design the joint configuration of the pre-grasping cage in each test. For terminal grasping cages, the joint configurations of the robotic arms are set as follows: for Test (a), $\boldsymbol{\theta}^{\mathrm{a}}=[44.92,-89.51,44.59]^{\mathrm{T}}\left({ }^{\circ}\right)$ and $\boldsymbol{\theta}^{\mathrm{b}}=[-47.82,89.34,-41.52]^{\mathrm{T}}\left({ }^{\circ}\right)$; for Test (b), $\quad \boldsymbol{\theta}^{\mathrm{a}}=[33.70,-63.91$, $30.21]^{\mathrm{T}}\left({ }^{\circ}\right)$ and $\boldsymbol{\theta}^{\mathrm{b}}=[-32.24,64.00,-31.76]^{\mathrm{T}}\left({ }^{\circ}\right)$; for Test (c), $\boldsymbol{\theta}^{\mathrm{a}}=[14.34,-52.71,38.38]^{\mathrm{T}}\left({ }^{\circ}\right)$ and $\boldsymbol{\theta}^{\mathrm{b}}=[-4.93,45.77,-40.84]^{\mathrm{T}}\left({ }^{\mathrm{o}}\right)$; for $\quad$ Test $\quad(\mathrm{d}), \quad \boldsymbol{\theta}^{\mathrm{a}}=[37.38,-72.36,34.98]^{\mathrm{T}}\left({ }^{\circ}\right)$ and $\boldsymbol{\theta}^{\mathrm{b}}=[-39.06,72.18, \quad-33.12]^{\mathrm{T}}\left({ }^{\circ}\right)$; and for Test (e), $\boldsymbol{\theta}^{\mathrm{a}}=[31.02,-71.97,40.95]^{\mathrm{T}}\left({ }^{\circ}\right)$ and $\boldsymbol{\theta}^{\mathrm{b}}=[-32.76,72.28,-39.52]^{\mathrm{T}}\left({ }^{\circ}\right)$. Here, the straight interpolation is used to plan joint trajectories from the pre-grasping cage to the terminal grasping cage in each test.

The snapshots in Fig. 15 show the dual-arm space robot simulator caging non-graspable objects. The rectangular and circular effector pairs capture non-graspable objects in the presence of shape uncertainties. The last snapshot in each test is captured after the support has been removed and each object remains in the robotic cage, despite the gravitational force. In total, ten independent tests are conducted to evaluate the task success rate of the proposed method and the results are listed in Table IV. Here, based on our analysis of the test processes, we conclude that the larger the distance difference $(\Delta d=d(\mathrm{P}, \mathrm{B})-d(\mathrm{G}, \mathrm{C}))$ is, the higher the capturing task success rate will be. For the failed tests, the main reasons are the effect of gravity (when we remove the bases, the equilibrium condition is not satisfied.) and asynchronous motions of the two arms (due to abnormal communication from the control system). In summary, the results demonstrate the superior performance of the proposed method in capturing non-graspable objects.

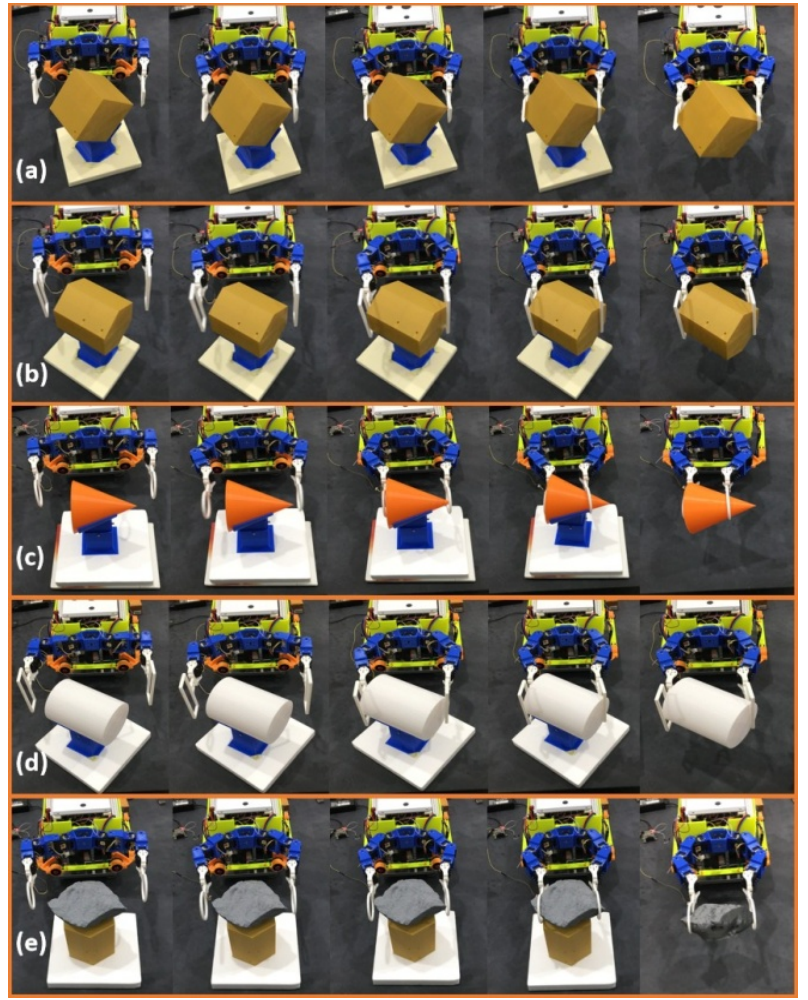

Fig. 15. Snapshots of caging tests

TABLE IV TEST RESULTS

\begin{tabular}{lcc}
\hline \hline \multicolumn{1}{c}{$\begin{array}{c}\text { Antipodal pair of the } \\
\text { object }\end{array}$} & Effector type & Success rate \\
\hline Test (a): vertex-vertex pair & Triangular effectors & $9 / 10$ \\
Test (b): edge-edge pair & Rectangular effectors & $8 / 10$ \\
Test (c): vertex-face pair & Circular effectors & $10 / 10$ \\
Test (d): edge-edge pair & Rectangular effectors & $7 / 10$ \\
Test (e): vertex-vertex pair & Circular effectors & $9 / 10$ \\
\hline \hline
\end{tabular}

\section{CONCLUSIONS AND FUTURE WORK}

In this study, we provide a new insight into the capture of non-graspable space objects, namely, objects without any graspable features. A caging-pair method is presented for capturing non-graspable space objects and the main strategy is to use a series of hollow-shaped effector pairs to form robotic cages for capturing objects. Due to its simple capturing principle and mechanism design, the proposed method has the intrinsic advantages of high robustness and reliability. For caging objects with a desired capture capability, the concept of caging compatibility, its corresponding index, and a planning algorithm are proposed for planning the near-optimal pre-grasping configurations of the dual-arm space robot. Ten independent simulation trials are conducted on five typical non-graspable objects and the analysis results demonstrate that the proposed planning algorithm is effective. A near-optimal solution can be obtained when the number of sampling points in the Monte Carlo algorithm is equal to or larger than 500. Accordingly, five groups of experimental tests are conducted to practically evaluate the proposed method using three types of hollow-shaped effector pairs. The experimental results demonstrate that the proposed method performs robustly and 
reliably on non-graspable objects, with an average success rate of $86 \%$ in the capturing tasks.

Furthermore, with the qualitative comparative analysis, the proposed method has the following advantages: 1) Compared with tether nets [18], [19], tether grippers [20], [21], and unit-type caging methods [22], the proposed end-effectors are affixed at the end of robotic arms to form a combined cage; hence, is a simpler and easier-to-implement caging method with high reusability and reliability. 2) For a robotic tentacle [23], it is difficult to capture the non-graspable object in Fig. 1(b3), whereas the proposed method easily handles that object with dexterous manipulability. 3) Compared with adhesive grippers [3], [17], the proposed end-effectors have a simpler mechanism design and they are robust and stable against the roughness and irregularity of objects in the space environment. In contrast, it is difficult for adhesive grippers to capture an object that has a rough surface, such as that in Fig. 1(b5).

Currently, our experiments are conducted in 2D space. Our end-effector prototypes have some limitations and the contact force is not considered in the capture task. In the future, we will explore an automatic replacement interface mechanism and the structure optimization of end-effectors and combine the proposed method with the compliant control using industrial robots equipped with force/torque sensors in 3D space.

\section{REFERENCES}

[1]. J. C. Liou, "An active debris removal parametric study for LEO environment remediation," Adv. Space Res., vol. 47, no. 11, pp. 1865-1876, 2011.

[2]. M. Shan, J. Guo, and E. Gill, "Review and comparison of active space debris capturing and removal methods," Prog. Aerosp. Sci., vol. 80, pp. 18-32, 2016.

[3]. H. Jiang, E. W. Hawkes, C. Fuller, M. A. Estrada, S. A. Suresh, N. Abcouwer, A.K. Han, S. Wang, C. J. Ploch, A. Parness, and M. R. Cutkosky, "A robotic device using gecko-inspired adhesives can grasp and manipulate large objects in microgravity," Sci Robot., vol. 2, no. 7, eaan4545, 2017.

[4]. G. Roesler, J. Paul, and H. Glen, "Orbital mechanics," IEEE Spectr., vol.54, no. 3, pp. 44-50, 2017.

[5]. L. Felicetti, P. Gasbarri, A. Pisculli, M. Sabatini, and G. B. Palmerini, "Design of robotic manipulators for orbit removal of spent launchers' stages," Acta Astronaut., vol. 119, pp.118-130, 2016.

[6]. K. Yoshida, H. Nakanishi, H. Ueno, N. Inaba, T. Nishimaki, and M. Oda, "Dynamics, control and impedance matching for robotic capture of a non-cooperative satellite," Adv Robot., vol. 18, no. 2, pp.175-198, 2004.

[7]. W. Xu, B. Liang, C. Li, and Y. Xu, "Autonomous rendezvous and robotic capturing of non-cooperative target in space," Robotica, vol. 28, no. 5 , pp.705-718, 2010.

[8]. X. Zhang and J. Liu, "Effective motion planning strategy for space robot capturing targets under consideration of the berth position," Acta Astronaut., vol. 148, pp. 403-416, 2018.

[9]. J. Artigas, D. M. Stefano, W. Rackl, and A. Albu-Schäeffer, "The OOS-SIM: An on-ground simulation facility for on-orbit servicing robotic operations," in Proc. IEEE Int. Conf. Robot. Autom., pp. $2854-2860,2015$.

[10]. S. A. Moosavian, R. Rastegari, and E. Papadopoulos, Multiple impedance control for space free-flying robots. J Guid Control Dyn., vol. 28, no. 5, pp. 939-947, 2005.

[11]. F. Aghili, "A prediction and motion-planning scheme for visually guided robotic capturing of free-floating tumbling objects with uncertain dynamics," IEEE Trans. Robot., vol. 28, no. 3, pp. 634-649, 2012.

[12]. R. A. Mccourt, and C. W. D. Silva, "Autonomous robotic capture of a satellite using constrained predictive control," IEEE/ASME Trans. Mechatronics., vol. 11, no. 6, pp. 699-708, 2006.
[13]. P. Huang, M. Wang, Z. Meng, F. Zhang, Z. Liu, and H. Chang, "Reconfigurable spacecraft attitude takeover control in post-capture of target by space manipulators," J Franklin Inst, vol. 353, no. 9, pp. 1985-2008, 2016.

[14]. E. Brown, N. Rodenberg, J. Amend, A. Mozeika, E. Steltz, M. R. Zakin, and H. M. Jaeger, "Universal robotic gripper based on the jamming of granular material". Proc. Nat. Acad. Sci., vol.107, no 44, pp.18809-18814. 2010.

[15]. D. Yoon and Y. Choi, "Underactuated Finger Mechanism Using Contractible Slider-Cranks and Stackable Four-Bar Linkages," IEEE/ASME Trans.Mechatronics., vol.22, no. 5, pp.2046-2057. 2017.

[16]. M. Abdeetedal and M.R. Kermani, "Grasp and Stress Analysis of an Underactuated Finger for Proprioceptive Tactile Sensing," IEEE/ASME Trans.Mechatronics., vol.23, no. 4, pp.1619-1629, 2018.

[17]. L. Savioli, G. Sguotti, A. Francesconi, F. Branz, J. Krahn, C. Menon, "Morphing electroadhesive interface to manipulate uncooperative objects," in Proc. SPIE Sensors and Smart Structures Technologies for Civil, Mechanical, and Aerospace Systems, vol. 9061, pp. 906129, 2014.

[18]. I. Sharf, B. Thomsen, E. M. Botta, and A. K. Misra, "Experiments and simulation of a net closing mechanism for tether-net capture of space debris," Acta Astronaut., vol. 139, pp. 332-343, 2017.

[19]. F. Zhang, and P. Huang, "Releasing dynamics and stability control of maneuverable tethered space net," IEEE/ASME Trans. Mechatronics., vol. 22 , no.2, pp. 983-993, 2017.

[20]. S. I. Nishida, S. Kawamoto, Y. Okawa, F. Terui, and S. Kitamura, "Space debris removal system using a small satellite," Acta Astronaut., vol. 65, pp. 95-102, 2009.

[21]. P. Huang, D. Wang, Z. Meng, F. Zhang, and Z. Liu, "Impact dynamic modeling and adaptive target capturing control for tethered space robots with uncertainties," IEEE/ASME Trans. Mechatronics., vol. 21, no. 5, pp.2260-2271, 2016.

[22]. M. Gates, B. Muirhead, B. Naasz, M. McDonald, D. Mazanek, S. Stich, C. Paul, and R. Jim, "NASA's Asteroid Redirect Mission concept development summary," in Proc. IEEE Aerosp Conf., pp. 1-13, 2015.

[23]. M. Wilde, S. K. Choon, I. Walker, and J. Near, "Using Tentacle Robots for Capturing Non-Cooperative Space Debris-A Proof of Concept," In AIAA Space and Astronautics Forum and Exposition, pp.1-10, 2017.

[24]. S. I. Nishida and S. Kawamoto, "Strategy for capturing of a tumbling space debris," Acta Astronaut., vol. 68, pp. 113-120, 2011.

[25]. W. Wan, B. Shi, Z. Wang, and R. Fukui, "Multirobot Object Transport via Robust Caging," IEEE Trans. Syst., Man, Cybern., Syst., in press.

[26]. S. A. Moosavian, and E. Papadopoulos, "Free-flying robots in space: an overview of dynamics modeling, planning and control," Robotica, vol. 25, no 5, pp. 537-547, 2007.

[27]. W. Xu, J. Peng, B. Liang, and Z. Mu, "Hybrid modeling and analysis method for dynamic coupling of space robots," IEEE Trans. Aerosp. Electron. Syst., vol. 52, no 1, pp. 85-98, 2016.

[28]. C. Carignan and D. Akin, "The reaction stabilization of on-orbit robots," IEEE Control Syst., vol. 20, no. 6, pp. 19-33, 2000.

[29]. H. Ueno, Y. Wakabayashi, Y. Ohkami, S. Matunaga, R. Hayashi, and T. Yoshida, "Ground testbed of a reconfigurable brachiating space robot," Adv Robot., vol. 14, no. 5, pp.355-358, 2000.

[30]. H. Fujii, K. Uchiyama, H. Yoneoka, and T. Maruyama, "Ground-based simulation of space manipulators using test bed with suspension system," J Guid Control Dyn., vol.19, no. 5, pp.985-991, 1996.

[31]. W. Xu, B. Liang, Y. Xu, C. Li, and W. Qiang, "A ground experiment system of free-floating robot for capturing space target," J Intell Robot Syst., vol. 48, no. 2, pp.187-208, 2007.

[32]. J. Kindracki, K. Tur, P. Paszkiewicz, Ł. Mężyk, Ł. Boruc, and P. Wolański, "Experimental research on low-cost cold gas propulsion for a space robot platform," Aerosp. Sci. Technol., vol., 62, pp.148-157, 2017.

[33]. X. Zhang, J. Liu, and J. Feng, "Experimental Platform of Space Robot Grasping Various Nonprehensile Targets," in Proc. 50th International Symposium on Robotics (ISR 2018), pp. 1-7, 2018.

[34]. A.Rodiguez, M. T. Mason, and S. Ferry, "From caging to grasping," The Int. J. Robot. Res., vol. 31, no. 7, pp.886-900, 2012.

[35]. J. Seo, M. Yim, and V. Kumar, "A theory on grasping objects using effectors with curved contact surfaces and its application to whole-arm grasping," Int. J. Robot. Res., vol. 35, no. 9, pp.1080-1102, 2016.

[36]. J. Lee, P. H. Chang, R. S. Jamisola, "Relative impedance control for dual-arm robots performing asymmetric bimanual tasks," IEEE Trans. Ind. Electron., vol. 61, no.7, pp.3786-3796, 2013.

[37]. A. Ajoudani, N. G. Tsagarakis, J. Lee, M. Gabiccini and A. Bicchi, 
"Natural redundancy resolution in dual-arm manipulation using configuration dependent stiffness (CDS) control," in Proc. IEEE Conf. ICRA, pp. 1480-1486, 2014.

[38]. L. Yan, H. Yuan, W. Xu, Z. Hu, and B. Liang, "Generalized Relative Jacobian Matrix of Space Robot for Dual-Arm Coordinated Capture," $J$ Guid Control Dyn., vol.41, no. 5, pp.1202-1208, 2018.

[39]. P. Bose, D. Bremner, and G. Toussaint, "All convex polyhedra can be clamped with parallel jaw grippers," Comput. Geom., vol.6, pp.291-302, 1996.

[40]. C. Smith, Y. Karayiannidis, L. Nalpantidis, X. Gratal, P. Qi, D. V. Dimarogonas, D. Kragic, "Dual arm manipulation-A survey," Robotics and Autonomous systems, vol. 60, no.10, pp.1340-1353, 2012.

[41]. T. Yoshikawa, "Manipulability of robotic mechanisms," Int. J. Robot. Res., vol. 4, no. 2, pp.3-9, 1985.

[42]. P. Chiacchio, S. Chiaverini, L. Sciavicco, and B. Siciliano, "Global task space manipulability ellipsoids for multiple-arm systems," IEEE Trans. Robot. Autom., vol. 7, no. 5, pp.678-685, 1991.

[43]. S. Lee, "Dual redundant arm configuration optimization with task-oriented dual arm manipulability," IEEE Trans. Robot. Autom., vol. 5, no. 1, pp.78-97, 1989.

[44]. H. A. Park, and C. G. Lee, "Dual-arm coordinated-motion task specification and performance evaluation," in Proc. IEEE Conf. IROS, pp. 929-936, 2016.

[45]. S. L. Chiu, "Task compatibility of manipulator postures," Int. J. Robot. Res., vol. 7, no. 5, pp.13-21,1988.

[46]. P. Corke, Robotics, Vision and Control, Heidelberg: Springer 2017.

Xin Zhang (S'19) is pursuing his Ph.D. degree in mechatronic engineering in the State Key Laboratory of Robotics, Shenyang Institute of Automation, Chinese Academy of Sciences, Shenyang, China. His research interests include space robots, modular reconfigurable robots, and their mechanism design, modeling, and motion planning and control.

Jinguo Liu (M'07-SM'18) received his Ph.D. degree in mechatronics from Shenyang Institute of Automation (SIA), Chinese Academy of Sciences (CAS) in 2007. Since January 2011, he has been a Full Professor with SIA, CAS. In addition, he has held the Assistant Director position of State Key Laboratory of Robotics since 2008 and the Associate Director position of Center for Space Automation Technologies and Systems since 2015. His research interests include bio-inspired robotics and space robotics. He has authored/coauthored four books, over one hundred papers and fifty patents in these areas. Dr. Liu services as an Editor of several journals such as IEEE Access, Mechanical Sciences, and Chinese Journal of Mechanical Engineering.

Jingkai Feng received his M.S. degree in mechanical engineering from Shenyang Institute of Automation (SIA), Chinese Academy of Sciences (CAS), Shenyang, China, in 2018. Currently, he is pursuing his Ph.D. degree in mechatronic engineering in SIA, CAS. His research focuses on the bionic drill for planetary sampling and detection.

Yuwang Liu (M'12) received his Ph.D. degree in robotics from Shenyang Institute of Automation (SIA), Chinese Academy of Sciences (CAS), in 2010. Currently, he is a Full Professor with SIA, CAS. As a project leader, he is involved in National Natural Science Foundation of China, National High-tech R\&D Program of China (863 Program), and Key Program of Chinese Academy of Sciences.

Zhaojie Jiu (M'08-SM'16) received the B.S. in automatic control and the M.S. in intelligent robotics both from Huazhong University of Science and Technology, China, and the Ph.D. degree in intelligent robotics at the University of Portsmouth, UK. He held a research appointment at the University College London, London, U.K., before he started his independent academic position at the University of Portsmouth, U.K., in 2012. His research interests include machine intelligence, pattern recognition, and their applications on human
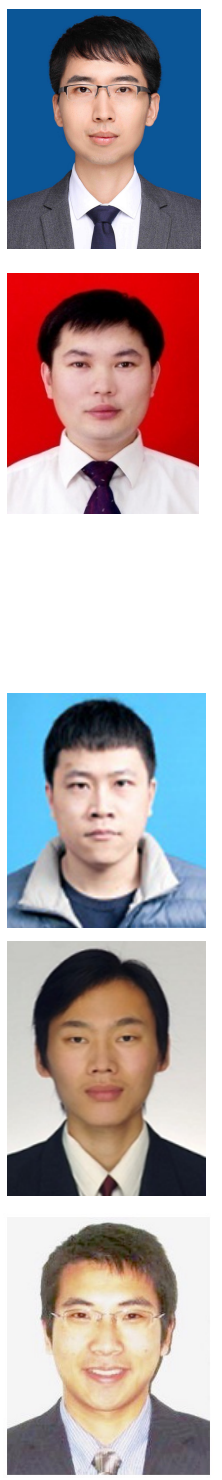
motion analysis. He has authored or co-authored over 170 publications in journals, book chapters, and conference proceedings and received four best paper awards and one Best AE Award in ICRA2018. Dr. Ju is an Associate Editor of the IEEE Transactions on Cybernetics, Journal of Intelligent \& Fuzzy Systems, and International Journal of Fuzzy Systems. 